\title{
Densidade do conjunto de endomorfismos com medida maximizante suportada em órbita periódica.
}

Juliano dos Santos Gonschorowski

TESE APRESENTADA

$\mathrm{AO}$

Instituto de Matemática e Estatística

DA

Universidade de SÃo Paulo

PARA

OBTENÇÃO DO TÍTULO

DE

Doutor EM CIÊNCIAS

Programa: Matemática Aplicada

Orientador: Prof. Dr. Fabio Armando Tal

Durante o desenvolvimento deste trabalho o autor recebeu auxílio financeiro do CNPq

São Paulo, fevereiro de 2012 



\title{
Densidade do conjunto de endomorfismos com medida maximizante suportada em órbita periódica.
}

\author{
Este exemplar corresponde à redação \\ final da tese devidamente corrigida \\ e defendida por Juliano dos Santos Gonschorowski \\ e aprovada pela Comissão Julgadora.
}

Banca Examinadora:

- Prof. Dr. Fabio Armando Tal (presidente) - IME-USP.

- Prof. Dr. Salvador Addas Zanata - IME-USP.

- Prof. Dr. Ricardo dos Santos Freire Junior - IME-USP.

- Prof. Dr. Artur Oscar Lopes - UFRGS.

- Prof. Dr. Eduardo Garibaldi - UNICAMP. 


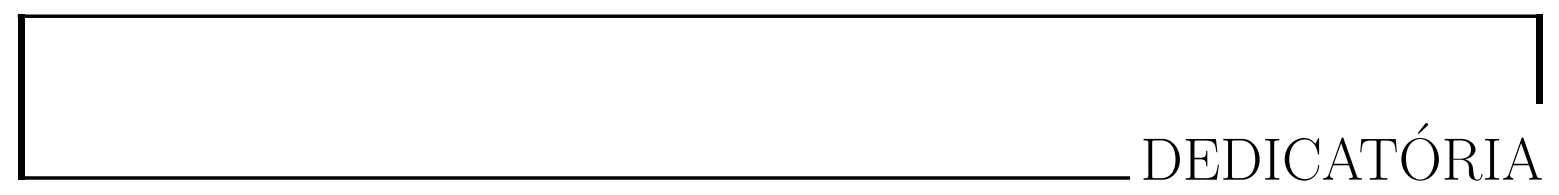

Aos meus pais Lourival e Marlene, e à minha esposa Graciele. 

Agradeço...

à minha esposa Graciele por estar ao meu lado em todos os momentos...

aos meus pais pelo amor, incentivo e apoio que sempre me deram...

ao meu orientador Fábio Tal, por quem tenho muita admiração, uma pessoa profissional e gente boa que me apresentou à teoria de otimização ergódica, e que sempre me ajudou nos momentos em que precisei...

à professora Helena que foi uma das responsáveis por eu ter entrado no doutorado na matemática aplicada...

à $\mathrm{CPG}$ e ao $\mathrm{CNPq}$ pelo apoio financeiro...

à minha amiga, Tatiane Batista por seu companheirismo durante o desenvolvimento da tese...

ao meus amigos Valentin e Cesar por sempre esclarecerem minhas dúvidas de matemática...

a todos os meus amigos do IME: Pricila, Ricardo, Bráulio, Débora, Dylene, Rose, Maysa, Fernanda, Andersons (o normal e o super), Oscar, Gustavo, Brunos (Hellboy e o Big), Naiára, Sebastian, Bernar, Humberto, Renata, Patrícia, Wonder e Marta pelos cafés no bloco A e pelo volei de sábado... se eu me esqueci de alguém, me perdoe... 

Demonstramos o seguinte teorema: Seja $M$ uma variedade Riemanniana compacta, conexa e sem bordo. Dados um endomorfismo $f: M \rightarrow M$, uma função contínua $\phi: M \rightarrow \mathbb{R} e \epsilon>0$, então existe um endomorfismo $\tilde{f}: M \rightarrow M$ tal que

$$
d(f, \tilde{f})=\max _{x \in M} d(f(x), \tilde{f}(x))<\epsilon
$$

e existe uma medida $\phi$-maximizante para $\tilde{f}$ que está suportada em uma órbita periódica. Este teorema é uma generalização dos resultados obtidos por S. Addas-Zanatta e F. Tal em [41].

Palavras-chave: medida maximizante, órbita periódica, endomorfismos. 

We prove the following theorem: Let $M$ be a bondaryless, compact and connected Riemannian Manifold. Given an endomorphism $f: M \rightarrow M$, a continuous function $\phi: M \rightarrow \mathbb{R}$ and $\epsilon>0$, then there exist an endomorphism $\tilde{f}: M \rightarrow M$ with

$$
d(f, \tilde{f})=\max _{x \in M} d(f(x), \tilde{f}(x))<\epsilon
$$

such that, some $\phi$-maximizing measure for $\tilde{f}$ is supported on a periodic orbit. This theorem is a generalization of the results [41] obtained by S. Addas-Zanatta and F. Tal.

Keywords: maximizing measure, periodic orbit, endomorphisms. 

Introdução

1 Preliminares 6

1.1 Teoria Ergódica . . . . . . . . . . . . . . . . . . . . . 6

1.2 Formalismo Termodinâmico . . . . . . . . . . . . . . . . 10

1.2.1 Entropia Métrica . . . . . . . . . . . . . . 11

1.2.2 Entropia Topológica . . . . . . . . . . . . . . 13

1.2.3 Pressão Topológica . . . . . . . . . . . . . . . . . 16

1.2.4 Estados de Equilíbrio . . . . . . . . . . . . . . 18

1.3 Sistemas Lagrangianos . . . . . . . . . . . . . . . . . . . . . . . . 19

1.3.1 Dinâmica Lagrangiana . . . . . . . . . . . . . . . . . . . . . . . 19

1.3.2 Valor Crítico de Mañé . . . . . . . . . . . . . . . . . . 21

2 Otimização Ergódica $\quad 24$

2.1 O conjunto das medidas maximizantes . . . . . . . . . . . . . 27

2.2 Alguns dos Principais Resultados Conhecidos . . . . . . . . . . . 28

3 Resultado Principal $\quad 34$

3.1 Definição do problema . . . . . . . . . . . . . . . . 34 
3.2 Caso $1 \ldots \ldots \ldots \ldots$

3.2 .1 Caso 1.1. . . . . . . . . . . . . . . . . . . . . . 39

3.2 .2 Caso 1.2. e Caso $2 \ldots \ldots$. . . . . . . . . . . . . . 40

3.3 O Retorno na Fronteira . . . . . . . . . . . . . . . . . . . . . 42

3.3 .1 A última perturbação . . . . . . . . . . . . . . . . . . . 45

3.4 Problemas Futuros . . . . . . . . . . . . . . . . . . . . 51

$\begin{array}{ll}\text { Bibliografia } & 56\end{array}$ 
A teoria ergódica e a teoria de otimização foram amplamente estudadas, porém separadamente. Os primeiros resultados ligando essas áreas apareceram a cerca de vinte anos atrás [28] [31] [32], e a apenas dez, começaram a se produzir trabalhos consolidando uma nova linha de pesquisa, atualmente chamada de Otimização Ergódica [9] [18] [19] [34]. Um dos fatores que explica o porquê desta teoria não ter se desenvolvido antes, é o fato de que as primeiras conjecturas foram intuídas com a ajuda de computadores $[18]$.

Sejam $\phi: X \rightarrow \mathbb{R}, f: X \rightarrow X$ funções contínuas e $X$ um espaço métrico (geralmente compacto). Em otimização ergódica estamos interessados em estudar o problema de maximizar o seguinte funcional $A_{\phi}: \mathcal{M}_{f} \rightarrow \mathbb{R}$

$$
A_{\phi}(\mu)=\int \phi d \mu
$$

onde $\mathcal{M}_{f}$ denota o espaço das medidas borelianas de probabilidade $f$-invariantes. Uma tal medida que maximiza o funcional $A_{\phi}$ é chamada $\phi$-maximizante. O conjunto das medidas $\phi$-maximizantes será denotado por $\mathcal{M}_{\phi-\max }$ ou $\mathcal{M}_{\max }(\phi)$.

Várias questões surgem deste problema:

1. Se fixarmos a dinâmica $f$ o que acontece com as medidas $\phi$-maximizantes quando variamos $\phi$ ? Ter suporte numa órbita periódica é uma propriedade genérica? 
2. Dada $f$ e $\mu$ uma medida ergódica $f$-invariante, existe uma $\phi$ contínua para a qual $\mu$ maximiza $A_{\phi}$ ? Caso exista podemos escolher $\phi$ diferenciável?

3. Se fixarmos a dinâmica $f$ podemos dizer que ter suporte total é uma propriedade genérica para as funções contínuas que possuem medidas $\phi$-maximizantes? E se $f$ for $C^{1}$ a generecidade acontece nesta topologia?

4. O que podemos fazer no caso em que o espaço métrico $X$ não é compacto?

5. O que acontece se fixamos a função $\phi$ e variarmos a dinâmica?

Para estas questões existem várias respostas parciais. Na questão (1) temos [18] [27] [35] e em particular T. Bousch, que em [7] provou que, fixada a dinâmica $f(x)=$ $2 x$ no círculo, para cada função $\rho_{\omega}(x)=\cos (2 \pi(x-\omega))$ existe uma única medida $\rho$-maximizante $\mu_{\omega}$ que tem suporte numa órbita periódica para todo $\omega \in \mathbb{S}^{1}$ exceto para um conjunto de medida nula e dimensão de Hausdorff zero.

Na questão (2) O. Jenkinson [21] provou que fixada uma dinâmica $f$ definida em um espaço métrico compacto $X$, então o conjunto $\cup_{\phi \in C^{0}(X)} \mathcal{M}_{\phi-\max }$, é precisamente o conjunto das faces fechadas de $\mathcal{M}_{f}$. Em particular dada uma medida ergódica em $\mathcal{M}_{f}$ ela é um ponto extremal e uma face fechada, assim existe uma $\phi \in C^{0}(X)$ tal que esta medida ergódica é a única medida $\phi$-maximizante.

Para a questão (3) O. Jenkinson [18] provou que, fixada a dinâmica $2 x$, para uma função genérica $\phi \in C^{0}$, toda medida $\phi$-maximizante possui suporte total.

Já para a questão (4) nem mesmo garantir a existência de medidas maximizantes é uma tarefa fácil [5] [22], especificamente em [19] e [20] O. Jenkinson et. al. trabalharam com subshifts do tipo finito sobre um alfabeto contável e encontraram condições necessárias para garantir a existência de formas normais, e assim poder caracterizar as medidas $\phi$-maximizantes em termos de seu suporte, mais precisamente eles mostraram que sob certas hipóteses $\mathcal{M}_{\phi-\max }=\left\{\mu \in \mathcal{M}_{f}: \operatorname{supp}(\mu) \subset \tilde{\phi}^{-1}(\sup (\tilde{\phi}))\right\}$, onde $\tilde{\phi}$ é a forma normal de $\phi$. Já em [6] R. Bissacot e R. Freire Jr. garantiram a existência 
de medidas maximizantes para shifts de Markov irredutíveis sob um alfabeto contável, assumindo que $\phi$ é coerciva e com variação limitada.

A questão (5) é um problema difícil já que, uma vez que mudamos a dinâmica mudamos os espaços das medidas, assim podemos criar novas medidas $\phi$-maximizantes e perder as que já tinhamos.

Dentre as questões apresentadas, é importante ressaltar que a questão (1) parece ter sido motivada por uma importante conjectura de Mañé [29], que diz que medidas minimizantes para fluxos Lagrangianos são genericamente suportadas em trajetórias periódicas. Nosso resultado principal é uma variação desta questão e da questão (5) e está sumarizado no seguinte teorema:

Teorema 0.0.1. Seja $M$ uma variedade Riemanniana compacta, conexa e sem bordo. Dados um endomorfismo $f: M \rightarrow M$, uma função contínua $\phi: M \rightarrow \mathbb{R} e \epsilon>0$, então existe um endomorfismo $\tilde{f}: M \rightarrow M$ tal que

$$
d(f, \tilde{f})=\max _{x \in M} d(f(x), \tilde{f}(x))<\epsilon
$$

tal que podemos encontrar uma medida $\phi$-maximizante para $\tilde{f}$ suportada em uma órbita periódica.

Este teorema é uma generalização dos resultados de S. Addas-Zanada e F. Tal que o provaram quando a variedade $M$ é o círculo [41]. Um resultado similar dos mesmos autores mostra o teorema quando as funções consideradas são homeomorfismos definidos em uma variedade Riemanniana compacta [42].

A tese esta dividida em três capítulos, no primeiro fazemos uma revisão sobre alguns conceitos de teoria ergódica, no segundo apresentamos alguns resultados sobre otimização ergódica que dizem respeito ao problema central. No terceiro capítulo realizamos a construção do endomorfismo $\tilde{f}$ que prova o teorema (0.0.1). 



\section{CAPÍTULO 1}

PRELIMINARES

\section{$1.1 \quad$ Teoria Ergódica}

Nesta secção apresentamos alguns resultados de teoria ergódica que serão utilizados no desenvolvimento do trabalho. Para maiores informações sobre o tema indicamos [43].

Definição 1.1.1. O suporte de uma medida $\mu \in \mathcal{M}_{f}(X)$ é o conjunto dos pontos $x \in X$ tal que para todo aberto $A$, com $x \in A$ temos $\mu(A)>0$. O conjunto $\mathcal{M}_{f}(X)$ é o conjunto das medidas borelianas de probabilidades $f$-invariantes sobre $X$, também denotado por $\mathcal{M}_{f}$, quando $X$ estiver fixado.

O exemplo a seguir é o de uma medida invariante com suporte em uma órbita periódica.

Exemplo 1.1.2. Suponha que $X$ é um espaço topológico e $f: X \rightarrow X$ contínua. Seja $\delta_{x} \quad a$ medida de Dirac associada a um ponto $x$ definida por: $\delta_{x}(A)=\left\{\begin{array}{l}1, \text { se } x \in A \\ 0, \text { se } x \notin A\end{array}\right.$. Então a medida

$$
\mu=\frac{1}{n} \sum_{j=0}^{n-1} \delta_{f^{j}(p)}
$$

é invariante por $f$ se, e somente se, $p$ é um ponto periódico de $f$ com período $n$. 


\section{$(\Rightarrow)$}

Supondo que a medida em questão é $f$-invariante e que os pontos $\left\{f^{i}(p)\right\}_{i=0}^{n-1}$ são dois a dois distintos temos:

$$
\mu\left(f^{-1}(p)\right)=\mu(p)
$$

mas neste caso $\frac{1}{n} \sum_{j=0}^{n-1} \delta_{f^{j}(p)}(p)=\frac{1}{n} \sum_{j=1}^{n} \delta_{f^{j}(p)}(p)$, portanto, $\delta_{f^{n}(p)}(p)=\delta_{p}(p)=1 \Rightarrow$ $f^{n}(p)=p$.

$(\Leftarrow)$

Seja $A \subset X$ um conjunto mensurável qualquer. Mostremos que $\mu(A)=\mu\left(f^{-1}(A)\right)$.

$$
\begin{aligned}
\frac{1}{n} \sum_{j=0}^{n-1} \delta_{f^{j}(p)}\left(f^{-1}(A)\right) & =\frac{1}{n} \#\left\{0 \leq j \leq n-1, f^{j}(p) \in f^{-1}(A)\right\} \\
& =\frac{1}{n} \#\left\{0 \leq j \leq n-1, f^{j+1}(p) \in A\right\} \\
& =\frac{1}{n} \#\left\{1 \leq j \leq n, f^{j}(p) \in A\right\} .
\end{aligned}
$$

Mas, como $f^{n}(p)=p$,

$$
\begin{aligned}
\frac{1}{n} \#\left\{1 \leq j \leq n, f^{j}(p) \in A\right\} & =\frac{1}{n} \#\left\{0 \leq j \leq n-1, f^{j}(p) \in A\right\} \\
& =\frac{1}{n} \sum_{j=0}^{n-1} \delta_{f^{j}(p)}(A) .
\end{aligned}
$$

A partir de agora vamos apresentar as propriedades de $M_{f}(X)$, quando $X$ é um espaço métrico compacto e $f: X \rightarrow X$ é uma transformação contínua. Mas antes vamos definir ergodicidade.

Definição 1.1.3. Uma medida $\mu \in \mathcal{M}_{f}(X)$ é chamada ergódica se para todo conjunto $A \in \mathcal{B}$ com $f^{-1}(A)=A$ temos uma das seguintes opções:

- $\mu(A)=0$;

- $\mu(A)=1$. 
A demonstração dos próximos dois teoremas pode ser encontrada em [37].

Teorema 1.1.4. (Representação de Riesz) Se X é um espaço métrico compacto e se $\xi: \mathcal{C}^{0}(X) \rightarrow \mathbb{R}$ é um funcional linear, contínuo, positivo definido sobre $\mathcal{C}^{0}(X)$ o espaço das funções contínuas de $X$ em $\mathbb{R}, \operatorname{com} \xi(1)=1$, então existe uma única probabilidade $\mu \in \mathcal{M}(X)$ tal que

$$
\int_{X} f d \mu=\xi(f)
$$

para toda $f \in \mathcal{C}^{0}(X)$.

Definição 1.1.5. Dado um espaço vetorial topológico normado $V$ e seu dual topológico $V^{*}$, definimos uma topologia em $V^{*}$ chamada fraca- ${ }^{*}$ da seguinte forma: $f_{i} \rightarrow 0 \Leftrightarrow$ $f_{i}(v) \rightarrow 0$ para todo $v \in V$. Equivalentemente podemos definir a topologia fraca- ${ }^{*}$ como a menor topologia em $V^{*}$ que torna os funcionais $J_{x}(f)=f(x)$ de $V^{* *}$ contínuos.

Teorema 1.1.6. (Banach-Alaoglu) Dado um espaço de Banach separável e compacto $E$, então a bola unitária no dual $\left\{g \in E^{*}:\|g\| \leq 1\right\}$ é compacta na topologia fraca- *

Uma consequência destes teoremas é o seguinte:

Teorema 1.1.7. Sejam $X$ um espaço métrico, compacto e $f: X \rightarrow X$ uma transformação contínua. Então $\mathcal{M}_{f}(X)$ é não vazio, além disso é um espaço métrico, compacto e convexo.

Dois teoremas fundamentais na teoria ergódica cuja demonstração pode ser encontrada em [43] e [24], são apresentados abaixo.

Teorema 1.1.8. (Recorrência de Poincaré) Sejam $(X, \mathcal{B}, \mu)$ espaço de probabilidade e $f: X \rightarrow X$ uma função mensurável que preserva $\mu$. Considere $E \in \mathcal{B}$ de medida positiva. Então para $\mu$-q.t.p $x \in E$ retorna infinitas vezes para $E$.

\footnotetext{
${ }^{1} \xi$ é dito positivo se para todo $f \in \mathcal{C}^{0}(X) \operatorname{com} f \geq 0$ temos $\xi(f) \geq 0$.
} 
Teorema 1.1.9. (Ergódico de Birkhoff) Seja $(X, \mathcal{B}, \mu)$ um espaço de probabilidade e $T: X \rightarrow X$ uma transformação que preserva medida. Então,

1. se $f \in \mathcal{L}^{1}(X)$, o limite

$$
\tilde{f}(x):=\lim _{n \rightarrow \infty} \frac{1}{n} \sum_{j=0}^{n-1} f\left(T^{j}(x)\right)
$$

existe para quase todo ponto $x \in X$.

2. $\tilde{f}$ é uma função invariante, ou seja, $\tilde{f}(x)=\tilde{f}(T(x))$ em quase todo ponto.

3. $\int_{X} \tilde{f} d \mu=\int_{X} f d \mu$.

4. $\lim _{n \rightarrow \infty}\left\|\tilde{f}-\frac{1}{n} \sum_{j=0}^{n-1} f \circ T^{j}\right\|_{1}=0$

Os próximos teoremas mostram que as medidas ergódicas são pontos extremais do convexo (que na verdade é um simplexo) $\mathcal{M}_{f}$ e qualquer medida neste espaço pode ser escrita como combinação de medidas ergódicas.

Teorema 1.1.10. Sejam $X$ um espaço métrico compacto e $f: X \rightarrow X$ contínua. Se $\mu$ é ergódica e $v \in \mathcal{M}_{f}(X)$ é absolutamente contínua ${ }^{1}$ com respeito a $\mu$ então, $v=\mu$.

Lema 1.1.11. Sejam $(X, \mathcal{B}, \mu)$ um espaço de medida e $f: X \rightarrow X$ contínua, onde $X$ é um espaço métrico compacto. Então $\mu$ é ergódica se, e somente se, $\mu$ é ponto extremal de $\mathcal{M}_{f}(X)$.

Demonstração. Suponha que $\mu$ é ergódica e $\mu=t \mu_{1}+(1-t) \mu_{2}$, com $0<t<1$ e $\mu_{1}, \mu_{2} \in \mathcal{M}_{f}(X)$. Então, como $\mu_{1}$ e $\mu_{2}$ são absolutamente contínuas com respeito a $\mu$, do teorema anterior segue que $\mu_{1}=\mu$ e $\mu_{2}=\mu$. Portanto, $\mu$ é ponto extremal de $\mathcal{M}_{f}(X)$.

\footnotetext{
${ }^{1}$ Uma medida $\mu \in \mathcal{M}_{f}$ é absolutamente contínua com respeito a $\nu \in \mathcal{M}_{j}$ se para todo conjunto $A$ $\operatorname{com} \nu(A)=0$ implicar $\mu(A)=0$.
} 
Suponha agora que $\mu$ não é ergódica. Então existe $A \in \mathcal{B}$ um conjunto invariante com $0<\mu(A)<1$. Observemos que para todo $B \in \mathcal{B}$,

$$
\begin{aligned}
& \mu(B)=\mu(A) \cdot \frac{\mu(B \cap A)}{\mu(A)}+\mu\left(A^{C}\right) \cdot \frac{\mu\left(B \cap A^{C}\right)}{\mu\left(A^{C}\right)} \\
& \mu(B)=\mu(A) \cdot \frac{\mu(B \cap A)}{\mu(A)}+(1-\mu(A)) \cdot \frac{\mu\left(B \cap A^{C}\right)}{\mu\left(A^{C}\right)} .
\end{aligned}
$$

Assim, basta tomar $\mu_{1}(B)=\frac{\mu(B \cap A)}{\mu(A)}$ e $\mu_{2}(B)=\frac{\mu\left(B \cap A^{C}\right)}{\mu\left(A^{C}\right)}, \forall B \in \mathcal{B}$.

Temos que $\mu_{1} \in \mathcal{M}_{f}(X)$ pois, usando que $f^{-1}(A)=A$,

$$
\mu_{1}\left(f^{-1}(B)\right)=\frac{\mu\left(f^{-1}(B) \cap A\right)}{\mu(A)}=\frac{\mu\left(f^{-1}(B \cap A)\right)}{\mu(A)}=\frac{\mu(B \cap A)}{\mu(A)}=\mu_{1}(B) .
$$

Analogamente $\mu_{2} \in \mathcal{M}_{f}(X)$.

Portanto, $\mu$ não é ponto extremal de $\mathcal{M}_{f}(X)$.

O próximo teorema é uma consequência do Teorema de Choquet, ambos podem ser encontrados em [24]. Ele garante que podemos escrever qualquer medida em $\mathcal{M}_{f}$ como uma combinação de medidas ergódicas que são extremais.

Teorema 1.1.12. (Decomposição Ergódica) Sejam X um espaço compacto e $f: X \rightarrow X$ uma função contínua. Então, para cada $\mu \in \mathcal{M}_{f}(X)$, existe $\rho_{\mu}$ uma medida definida sobre os borelianos do espaço métrico compacto $\mathcal{M}_{f}(X)$, com a propriedade que $\rho_{\mu}\left(\operatorname{EM}_{f}(X)\right)=1 e$

$$
\mu=\int_{E \mathcal{M}_{f}(X)} m d \rho_{\mu}(m),
$$

onde $E \mathcal{M}_{f}(X)$ ou $\mathcal{M}_{\text {erg }}$ denotam o conjunto das medidas ergódicas em $\mathcal{M}_{f}(X)$.

\subsection{Formalismo Termodinâmico}

Segundo D. Ruelle [38], em mecânica estatística um dos principais problemas estudados é a transição de fase, por exemplo quando diminuímos gradativamente a temperatura 
da água, suas propriedades mudam de maneira suave, porém no momento que ela congela a mudança é drástica. Nesta secção apresentamos algumas definições e resultados do formalismo termodinâmico para que no próximo capítulo possamos falar das motivações do surgimento da otimização ergódica. Para maiores informações sobre o tema indicamos [38]. As demonstrações dos resultados sobre entropia e princípio variacional, podem ser encontrados em [43].

\subsubsection{Entropia Métrica}

Vamos definir a entropia métrica de uma transformação logo depois de definirmos a entropia de uma partição. Seja $(X, \mathcal{B}, \mu)$ um espaço de probabilidade sobre a sigma álgebra dos borelianos e $\xi=\left\{A_{1}, \ldots, A_{k}\right\}$ uma partição de $X$ (por definição numa partição os conjuntos são dois a dois disjuntos e sua união é $X$ ).

Definição 1.2.1. A entropia de uma partição $\xi$ é denotada por

$$
H(\xi)=H_{\mu}(\xi)=-\sum_{j=1}^{k} \mu\left(A_{j}\right) \ln \mu\left(A_{j}\right) .
$$

A entropia da partição é a quantidade média de informação trazida por um experimento e cujos resultados possíveis são indistinguiveis nos elementos da partição. Por exemplo, podemos considerar os 36 resultados possíveis de um lançamento de dois dados, e definir uma partição $\xi=A_{2}, A_{3}, \ldots, A_{12}$ onde $A_{j}$ representa o conjunto dos resultados que somam $j$. Assim, $\mu\left(A_{7}\right)=\frac{6}{36}, \mu\left(A_{6}\right)=\mu\left(A_{8}\right)=\frac{5}{36}, \ldots, \mu\left(A_{2}\right)=\mu\left(A_{12}\right)=\frac{1}{36}$. A entropia desta partição é igual a $2,33 \ldots$.

Podemos estudar a informação proveniente de se conhecer a trajetória da órbita futura de $x$ por uma transformação $T$ que preserva medida nos elementos de uma partição. Seja $i_{n}$ a representação do conjunto da partição

$$
T^{-n} \xi=\left\{T^{-n} A_{1}, \ldots, T^{-n} A_{k}\right\}
$$

ao qual $x$ pertence. Se tivermos $i_{0}$ e $i_{1}$ saberemos em quais elementos de $\xi$ o ponto $x$ e sua iterada $T x$ estão. Toda essa informação poderia ser fornecida por uma única 
partição, dada por todas as intersecções possíveis entre um elemento de $\xi$ e um elemento de $T^{-1} \xi$. Essa partição é denotada por $\xi \vee T^{-1} \xi$. Assim os elementos da partição

$$
\xi^{n}=\xi \vee T^{-1} \xi \vee \ldots \vee T^{-n+1} \xi
$$

representam todas as possibilidades de trajetórias sobre os elementos de $\xi$, em $n$ etapas.

A entropia de $\xi^{n}$ mede a informação média obtida com a determinação das $n$ primeiras posições de uma trajetória, outra informação interessante é a média da entropia por unidade de tempo.

Definição 1.2.2. Define-se entropia de $T$ com respeito à partição $\xi$ como sendo

$$
h(T, \xi)=h_{\mu}(T, \xi)=\lim _{n \rightarrow \infty} \frac{1}{n} H\left(\xi^{n}\right) .
$$

Podemos mostrar que esse limite existe usando o lema (1.2.3) e a propriedade da entropria de duas partições resultante do lema (1.2.4):

$$
H(\xi \vee \eta) \leq H(\xi)+H(\eta)
$$

Definimos a entropia de $T$, denontado por $h_{\mu}(T)$ como sendo:

$$
h_{\mu}(T)=\sup _{\xi} h(T, \xi) .
$$

Lema 1.2.3. Seja $\left\{a_{n}\right\}$ uma sequência de números reais satisfazendo $a_{n+m} \leq a_{n}+a_{m}$ (subaditiva), para todo par $n, m$. Então $\lim _{n \rightarrow \infty} \frac{1}{n} a_{n}$ existe e é igual a $\inf _{n} \frac{1}{n} a_{n}$.

Demonstração. Seja $k \geq 1$ qualquer, escrevendo $n=j k+r$, com $r=0,1, \ldots, k-1$. Então

$$
\frac{a_{n}}{n}=\frac{a_{j k+r}}{j k+r} \leq \frac{a_{j k}}{j k+r}+\frac{a_{r}}{j k+r} \leq \frac{a_{j k}}{j k}+\frac{M}{n},
$$

onde $M=\max _{0 \leq r<k} a_{r}$, mais ainda,

$$
\frac{a_{n}}{n} \leq \frac{j a_{k}}{j k}+\frac{M}{n}=\frac{a_{k}}{k}+\frac{M}{n} .
$$

Portanto

$$
\limsup _{n} \frac{a_{n}}{n} \leq \frac{a_{k}}{k}
$$


para todo $k \geq 1$. Então

$$
\limsup _{n} \frac{a_{n}}{n} \leq \inf _{k} \frac{a_{k}}{k} \leq \liminf _{k} \frac{a_{k}}{k}
$$

logo limsup e liminf são iguais.

Lema 1.2.4. A função $g(x)=-x \ln x$, definida em $[0,1]$, com $g(0)=0$ por continuidade, é côncava. ${ }^{1}$

\subsubsection{Entropia Topológica}

\section{Definição original}

Seja $X$ um espaço topológico compacto. Denotamos por $\alpha, \beta, \gamma, \ldots$ as coberturas formadas por abertos de $X$ e por $\alpha \vee \beta$ a cobertura pelos conjuntos $A \cap B$, onde $A \in \alpha$ e $B \in \beta$. Usaremos a notação $\bigvee_{i=1}^{n} \alpha_{i}$ para indicar $\alpha_{1} \vee \ldots \vee \alpha_{n}$.

Dizemos que $\beta$ é um refinamento de $\alpha$, denotado por $\alpha \leq \beta$, se para todo $B \in \beta$ existir $A \in \alpha$ tal que $B \subset A$. Assim, sempre teremos $\alpha \leq \alpha \vee \beta$ e se $\beta$ for uma subcobertura de $\alpha$ então $\alpha \leq \beta$. Se $T: X \rightarrow X$ for uma transformação contínua, então $T^{-1} \alpha$, formada pelos conjuntos $T^{-1} A, A \in \alpha$, será também uma cobertura aberta de $X$. Demonstra-se facilmente que $T^{-1}(\alpha \vee \beta)=T^{-1} \alpha \vee T^{-1} \beta$ e que se $\alpha \leq \beta$ então $T^{-1} \alpha \leq T^{-1} \beta$. Definiremos $N(\alpha)$ como a menor cardinalidade entre as subcoberturas de $\alpha$. Como $X$ é compacto esse número sempre é finito, e maior ou igual a 1 (de fato, ele só pode ser igual a 1 se $X$ for um elemento de $\alpha$ ). A entropia topológica de $\alpha$ será dada por

$$
H(\alpha)=\ln N(\alpha)
$$

Imediatamente notamos que $H(\alpha)$ é um número não-negativo, igual a zero se e somente se $X \in \alpha$, que $\alpha \leq \beta$ implica $H(\alpha) \leq H(\beta)$, e que $H(\alpha \vee \beta) \leq H(\alpha)+H(\beta)$. Além disso,

\footnotetext{
${ }^{1}$ Uma função $f:[a, b] \rightarrow \mathbb{R}$ é chamada côncava se para qualquer $x, y \in[a, b]$ temos $f(x t+(1-t) y) \geq$ $t f(x)+(1-t) f(y)$, onde $t \in[0,1]$.
} 
$H\left(T^{-1} \alpha\right) \leq H(\alpha)$, mas se $T$ for sobrejetiva então $H\left(T^{-1} \alpha\right)=H(\alpha)$. Analogamente à definição de entropia de uma medida, podemos mostrar, supondo $T$ contínua, que

$$
\lim _{n \rightarrow \infty} \frac{1}{n} H\left(\bigvee_{i=0}^{n-1} T^{-i} \alpha\right)
$$

existe, bastando ver que

$$
a_{n}=H\left(\bigvee_{i=0}^{n-1} T^{-i} \alpha\right)
$$

satisfaz $a_{n+k} \leq a_{n}+a_{k}$, para quaisquer $k, n \geq 1$ (usa-se que $H(\alpha \vee \beta) \leq H(\alpha)+H(\beta)$ e que $\left.H\left(T^{-1} \alpha\right) \leq H(\alpha)\right)$. Esse limite é definido como a entropia de $T$ relativa a $\alpha$, denotada por $h(T, \alpha)$. É possível mostrar que $h(T, \alpha)$ é um número não-negativo, que $\alpha \leq \beta$ implica $h(T, \alpha) \leq h(T, \beta)$ e que $h(T, \alpha) \leq H(\alpha)$. Finalmente definimos, para uma transformação contínua $T$, sua entropia topológica, denotada por $h(T)$, como sendo o supremo dos valores $h(T, \alpha)$, tomados entre todas as coberturas abertas de $X$. Evidentemente o supremo pode ser tomado entre as coberturas finitas. A entropia topológica de $T$ é sempre um número não-negativo (ou infinito). Outras propriedades que podem ser verificadas são que, se $T$ é a transformação identidade, então $h(T)=0$ e que se $Y \subset X$ é um conjunto invariante fechado então $h(T \mid Y) \leq h(T)$.

Teorema 1. Se Té homeomorfismo então $h(T)=h\left(T^{-1}\right)$.

O seguinte teorema mostra que a entropia topológica é um invariante topológico.

Teorema 2. Sejam $X_{1}$ e $X_{2}$ espaços topológicos compactos, $T_{1}: X_{i} \rightarrow X_{i}, i=1,2$, transformações contínuas, e $\phi: X_{1} \rightarrow X_{2}$ uma função contínua e sobrejetiva tal que $\phi T_{1}=T_{2} \phi$. Então $h\left(T_{1}\right) \geq h\left(T_{2}\right)$. Se $\phi$ for homeomorfismo, então $h\left(T_{1}\right)=h\left(T_{2}\right)$.

Seja $l(\alpha)$ número de Lebesgue da cobertura $\alpha$, isto é, um número $l$ tal que qualquer conjunto de diâmetro menor do que $l$ está contido em algum elemento da cobertura. Pode-se mostrar que sempre existe um tal número. Evidentemente, se $\operatorname{diam}(\alpha)$ for menor do que o número de Lebesgue de $\gamma$ então $\gamma \leq \alpha$. O teorema seguinte pressupõe que $X$ esteja munido de uma métrica para estabelecer uma forma mais prática de calcular a entropia topológica usando coberturas abertas. 
Teorema 3. Sejam $(X, d)$ um espaço métrico compacto e T uma transformação contínua. Se $\left\{\alpha_{n}\right\}_{n \geq 1}$ é uma sequência de coberturas abertas com diâmetro indo a zero então

$$
\lim _{n \rightarrow \infty} h\left(T, \alpha_{n}\right)=h(T),
$$

bem entendido que os dois lados podem valer $\infty$.

\section{Definição de Bowen}

Sejam $(X, d)$ um espaço métrico compacto e $f: X \rightarrow X$ uma aplicação contínua. Para $n \in \mathbb{N}$ definimos a métrica $d_{n}$ em $X$ por $d_{n}(x, y)=\max _{k=0, \ldots, n-1} d\left(f^{k}(x), f^{k}(y)\right)$. Sejam $B_{n}(x ; r)=\left\{y \in X: d_{n}(x, y)<r\right\}$ e $\bar{B}_{n}(x ; r)$ seu fecho, temos que:

$$
\begin{aligned}
B_{n}(x ; r) & =\left\{y \in X: \max \left\{d(x, y), \ldots, d\left(f^{n-1} x, f^{n-1} y\right)\right\}<r\right\} \\
& =\bigcap_{i=0}^{n-1} f^{-i} B\left(f^{i} x ; r\right),
\end{aligned}
$$

onde $B\left(f^{i} x ; r\right)$ é a bola aberta com respeito a métrica $d$. Isto significa que dois pontos estão $r$-próximos segundo a métrica $d_{n}$ se seus primeiros $n$ iterados estiverem $r$-próximos segundo a métrica $d$.

Definição 1.2.5. Diz-se que $Y \subset X$ é um $(n, \epsilon)$-gerador se para todo $x \in X$ existir $y \in Y \operatorname{com}_{n}(x, y) \leq \epsilon$.

- Em outras palavras, $Y$ é um $(n, \epsilon)$-gerador se

$$
X=\bigcup_{y \in Y} \bigcap_{i=0}^{n-1} f^{-i} \bar{B}\left(f^{i} y ; \epsilon\right)
$$

Definimos por

$$
r_{n}(\epsilon)=\min \{\# Y: Y \text { é }(n, \epsilon) \text {-gerador }\}
$$

Usando a compacidade de $X$ tiramos que $r_{n}(\epsilon)$ é finito. De fato, seja $\left\{U_{1}, \ldots, U_{m}\right\}$ uma cobertura finita de $X$ com $\operatorname{diam}\left(U_{i}\right)<\epsilon$ para todo $i=1, \ldots, m$. Agora tomando um ponto em cada conjunto não-vazio da forma

$$
\bigcap_{k=0}^{n-1} f^{-k} U_{i_{k}}
$$

teremos um conjunto $(n, \epsilon)$-gerador, e há no máximo $m^{n}$ conjuntos deste tipo. 
Definição 1.2.6. A entropia topológica de $T$ denotada por $h_{\tau}(T)$ é dada por

$$
h_{\tau}(T)=\lim _{\epsilon \rightarrow 0} r(\epsilon, T) \text {, }
$$

onde $r(\epsilon, T)=\limsup _{n \rightarrow \infty} \frac{1}{n} \ln r_{n}(\epsilon)$.

Um importante resultado clássico relacionando as entropias métrica e topológica é o seguinte (retirado de [24]):

Teorema 1.2.7. (Princípio Variacional) Seja $f: X \rightarrow X$ uma função contínua do espaço métrico compacto $X$. Então vale a igualdade:

$$
h_{\tau}(f)=\sup _{\mu \in \mathcal{M}_{f}} h_{\mu}(f)
$$

\subsubsection{Pressão Topológica}

Vamos agora definir uma generalização do conceito de entropia topológica, introduzindo o conceito de pressão topológica de uma função $\phi: X \rightarrow \mathbb{R}$ com respeito a transformação $f: X \mapsto X$. Esse conceito (oriundo da Física, como o próprio nome indica) será também um invariante topológico de $f$, no sentido de que duas transformações topologicamente conjugadas ${ }^{1}$ atribuem a mesma pressão a uma função $\phi$. Vamos supor que o espaço $X$ é compacto para que as quantidades tratadas sejam sempre finitas, mas os conceitos expostos são de caráter geral não dependem desta condição.

Definição 1.2.8. Dado $n \in \mathbb{N}$ vamos denotar $S_{n} f(x)=\sum_{i=0}^{n-1} \phi\left(f^{i}(x)\right)$. Dado um conjunto $C \subset X$ denotamos $\phi_{n}(C)=\max _{x \in C} S_{n} f(x)$.

Dada uma cobertura $\alpha$ de $X$ definimos a pressão de $\phi$ com respeito a cobertura $\alpha$ como:

$$
P(\phi, \alpha)=\lim _{n \rightarrow+\infty} \frac{1}{n} \ln \inf _{\mathcal{U} \subset \alpha^{n}}\left\{\sum_{U \in \mathcal{U}} e^{\phi_{n}(U)}\right\},
$$

\footnotetext{
${ }^{1}$ Duas dinâmicas $f$ e $g$ são topologicamente conjugadas se existe um homeomorfismo $h$ tal que $f=h^{-1} \circ g \circ h$
} 
onde o ínfimo é tomado sobre todas as subcoberturas $\mathcal{U}$ de $\alpha^{n}{ }^{1}$

Definição 1.2.9. A pressão $P(\phi, f)$ de $\phi$ com respeito a $f$ é o supremo dos valores de $P(\phi, \alpha)$ sobre todas as coberturas abertas de $X$.

Proposição 1.2.10. Sejam $\phi, \psi \in C^{0}(X)$ e $C \in \mathbb{R}$. Então:

1) $P(0, f)=h_{\tau}(f)$

2) $P(\phi+C, f)=P(\phi, f)+C$

3) Se existir função limitada $u: X \rightarrow \mathbb{R}$ tal que $\phi$ se escreve como $\phi=\psi+u \circ f-u(e$ neste caso diremos que a função $\phi$ é cohomóloga a $\psi)$, então $P(\phi, f)=P(\psi, f)$.

Denotaremos também a pressão da medida $\mu$ com respeito a $\phi$ como sendo o número $P_{\mu}(\phi)=h_{\mu}(f)+\int \phi d \mu$. Para simplificar nossa notação, sempre que $f$ estiver fixada e não houver perigo de confusão, utilizaremos $P(\phi)$ para indicar $P(\phi, f)$. Note que se $t \in[0,1]$ e $\mu_{1}, \mu_{2} \in \mathcal{M}_{f}$ então

$$
P_{t \mu_{1}+(1-t) \mu_{2}}(\phi)=t P_{\mu_{1}}(\phi)+(1-t) P_{\mu_{2}}(\phi)
$$

Se tomarmos $\phi=0$ então a pressão de $\phi$ é exatamente a entropia topológica (pelo item 1 do teorema anterior). Podemos nos perguntar se a pressão topológica de um potencial contínuo qualquer admite uma caracterização via supremo no conjunto de medidas. A resposta para esta pergunta é sim e forma o importante teorema conhecido como Princípio Variacional para a pressão [24]:

Teorema 1.2.11. (Princípio Variacional para a Pressão) Se $\mathcal{M}_{f}$ é o conjunto das probabilidades invariantes para uma transformação contínua $f: X \rightarrow X$ do espaço compacto $X$ e $\phi: X \rightarrow \mathbb{R}$ é contínua, então

$$
P(\phi, f)=\sup _{\mu \in \mathcal{M}_{f}} P_{\mu}(\phi)=\sup _{\mu \in \mathcal{M}_{f}}\left\{h_{\mu}(f)+\int \phi d_{\mu}\right\}
$$

${ }^{1}$ Denotamos $\alpha^{n}=\alpha \vee \ldots \vee f^{-n+1} \alpha$ 


\subsubsection{Estados de Equilíbrio}

Uma importante classe de medidas invariantes são as chamadas estados de equilíbrio, que são definidas através de um princípio variacional e tem forte motivação física [38].

Definição 1.2.12. Uma medida $f$-invariante $\mu_{0}$ é dita um estado de equilíbrio para o potencial $\phi$ se

$$
P(\phi, f)=\sup _{\mu \in \mathcal{M}_{f}}\left\{h_{\mu}(f)+\int \phi d_{\mu}\right\}=h_{\mu_{0}}(f)+\int \phi d_{\mu_{0}} .
$$

Podemos nos perguntar que hipóteses a dinâmica deve satisfazer para que sempre exista pelo menos um estado de equilíbrio. A resposta está em definir alguns casos, como por exemplo, a ampla classe de funções chamadas expansivas [24]. Consideremos $X$ um espaço métrico compacto, salvo algumas excessões que serão apontadas no texto.

Definição 1.2.13. Uma transformação $f: X \rightarrow X$ é dita expansiva se existir uma constante $\epsilon_{0}$ (constante de expansividade) tal que: dados $x, y \in X$ com $x \neq y$, então existe $n \in \mathbb{N}$ tal que $d\left(f^{n}(x), f^{n}(y)\right) \geq \epsilon_{0}$.

Definição 1.2.14. Uma transformação diferenciável $f: X \rightarrow X$ é dita expansora se existir uma constante $\sigma>1$ tal que,

$$
\|D f(x) v\| \geq \sigma\|v\|
$$

para todo $x \in X$ e $v \in T_{x} X$.

Lema 1.2.15. Se $f$ é expansora então $f$ é expansiva.

Através da propriedade de expansividade é possível conectar o crescimento do números de pontos periódicos de $f$ com sua entropia topológica. Primeiro denotemos por $\operatorname{Per}(n)=\left\{x \in X: f^{n}(x)=x\right\}$, o conjunto de pontos periódicos de $X$ de período $n$, temos a seguinte proposição:

Proposição 1.2.16. Se $f$ é expansiva então

$$
\limsup \frac{1}{n} \ln \# \operatorname{Per}(n) \leq h_{\tau}(f) .
$$


A existência de estados de equilíbrios é dada pelo seguinte teorema:

Teorema 1.2.17. Seja $f: X \rightarrow X$ expansiva onde, $X$ é uma variedade compacta. Então para qualquer $\phi: X \rightarrow \mathbb{R}$ contínua, existe algum estado de equilíbrio para $\phi$.

A demonstração deste teorema que pode ser vista com detalhes em [24] segue do seguinte lema:

Lema 1.2.18. Dada $\phi: X \rightarrow \mathbb{R}$ contínua, a função $\mu \mapsto P(\mu)=h_{\mu}(f)+\int \phi d_{\mu}$ é semicontínua superiormente, isto é, dado $\epsilon>0$, existe $\delta>0$ tal que $\left|\mu-\mu_{0}\right|<\delta \Rightarrow$ $P(\mu)<P\left(\mu_{0}\right)+\epsilon$.

Além disso temos:

Teorema 1.2.19. Seja $f: X \rightarrow X$ expansiva onde, $X$ é uma variedade compacta. Então genericamente para $\phi: X \rightarrow \mathbb{R}$ contínua o estado de equilíbrio é único.

Este teorema foi estendido à transformações expansoras por Ruelle para maiores informações olhar [38].

\subsection{Sistemas Lagrangianos}

Uma introdução a Otimização Ergódica não seria completa sem mencionarmos os sistemas Lagrangianos devido seu papel histórico no desenvolvimento deste tema. A teoria aqui resumidamente apresentada, foi baseada no trabalho de G. Contreras e R. Iturriaga [12].

\subsubsection{Dinâmica Lagrangiana}

Seja $M$ uma variedade riemanianna $n$-dimensional sem bordo completa.

Definição 1.3.1. Uma curva $\gamma:[0, t] \rightarrow M$ é dita absolutamente contínua se, dado $\epsilon>0$ existe $\delta>0$ tal que para cada família $\left\{\left(a_{i}, b_{i}\right): i=1, \ldots, N\right\}$ de subintervalos de $[0, t]$ disjuntos dois a dois satisfazendo $\sum_{i=1}^{N}\left(b_{i}-a_{i}\right)<\delta$, tivermos que $\sum_{i=1}^{N} d_{M}\left(\gamma\left(b_{i}\right), \gamma\left(a_{i}\right)\right)<\epsilon$. 
Nem toda curva contínua é absolutamente contínua, por exemplo a função escadaria de Cantor no intervalo [0,1] é contínua mas não é absolutamente contínua [24].

Definição 1.3.2. Um Lagrangiano (autônomo) em $M$ é uma função $L: T M \rightarrow \mathbb{R} d e$ classe $C^{r}, r \geq 3$, satisfazendo as seguintes condições

i) Convexidade: L é estritamente convexo nas fibras, ou seja, para cada $x \in M e$ todos $v, w \in T_{x} M$ tem-se

$$
L(x, t v+(1-t) w)<t L(x, v)-(1-t) L(x, w), \forall t \in(0,1)
$$

ii) Superlinearidade: $L$ é superlinear, isto é, dado $A>0$ existe $B>0$ tal que

$$
L(x, v) \geq A\|v\|-B, \forall(x, v) \in T M
$$

iii) Limitação: Para todo $r \geq 0$,

$$
\begin{gathered}
l(r)=\sup _{(x, v) \in T M,|v| \leq r} L(x, v)<+\infty \\
g(r)=\sup _{|w|=1,|(x, v)| \leq r} w \cdot L_{v v}(x, v) \circ w<+\infty .
\end{gathered}
$$

A condição ii) é equivalente a dizer que a Hessiana $\frac{\partial^{2} L}{\partial v_{i} \partial v_{j}}(x, v)$ calculada em coordenadas lineares nas fibras $T_{x} M$, é uniformemente positiva definida para todo $(x, v) \in$ $T M$, i.e., existe $A>0$ tal que

$$
w \cdot L_{v v}(x, v) \cdot w \geq A|w|^{2}
$$

para todo $(x, v) \in T M$ e $w \in T_{x} M$.

A equação de Euler-Lagrange associada ao lagrangiano $L$ é:

$$
\frac{d}{d t} \frac{\partial L}{\partial v}(x, \dot{x})=\frac{\partial L}{\partial x}(x, \dot{x}) .
$$

A condição (iii) implica que a equação de Euler-Lagrange define um fluxo completo $\phi_{t}$ em $T M, \phi_{t}\left(x_{0}, v_{0}\right)=\left(x_{v}(t), \dot{x}_{v}(t)\right)$, onde $x_{v}: \mathbb{R} \rightarrow M$ é a solução da equação com $x_{v}(0)=x_{0}$ e $\dot{x}_{v}(0)=v_{0}$. 
Definição 1.3.3. Se L é um Lagrangiano em $M$ e $\gamma:[0, T] \rightarrow M$ é uma curva absolutamente contínua, definimos a ação $A_{L}(\gamma)$ de $\gamma$ por

$$
A_{L}(\gamma)=\int_{0}^{T} L(\gamma(t), \dot{\gamma}(t)) d t
$$

Um dos principais problemas em cálculo das variações é encontrar e estudar as curvas que minimizam a ação $A_{L}$. J. Mather provou em [31] que, quando o fluxo lagrangiano é completo, toda curva absolutamente contínua que minimiza a ação é $C^{2}$ e satisfaz a equação de Euler-Lagrange.

\subsubsection{Valor Crítico de Mañé}

Todas as curvas nesta secção serão consideradas absolutamente contínuas.

Definição 1.3.4. Para $x, y \in M$, seja

$$
\boldsymbol{C}(x, y)=\{\gamma:[0, T] \rightarrow M: T>0, \gamma(0)=x, \gamma(T)=y\}
$$

Para $k \in \mathbb{R}$ definimos o potencial da ação $\Phi_{k}: M \times M \rightarrow \mathbb{R} \cup\{-\infty\}$, por

$$
\Phi_{k}(x, y)=\inf _{\gamma \in C(x, y)} A_{L+k}(\gamma)
$$

Definição 1.3.5. Definimos o valor crítico $c=c(L)$ como:

$$
c(L)=\sup \left\{k \in \mathbb{R}: \exists \text { curva fechada } \gamma \operatorname{com} A_{L+k}(\gamma)<0\right\} .
$$

Observe que a função $k \longmapsto \Phi_{k}(x, y)$ é crescente. A superlinearidade implica que $L$ é limitada inferiormente. Como existe $k \in \mathbb{R}$ tal que $L+k \geq 0$, então $c(L)<+\infty$. Já que $k \longmapsto A_{L+k}(\gamma)$ é crescente para qualquer $\gamma$, temos que

$$
c(L)=\inf \left\{k \in \mathbb{R}: A_{L+k}(\gamma) \geq 0 \forall \text { curva fechada } \gamma\right\}
$$

Proposição 1.3.6. Temos que:

1. Para $k<c(L), \Phi_{k}(x, y)=-\infty$, para todo $x, y \in M$. 
2. Para $k \geq c(L)$ :

a) $\Phi_{k}(x, z) \leq \Phi_{k}(x, y)+\Phi_{k}(y, z)$, para todo $x, y \in M$.

b) $\Phi_{k}(x, x)=0$, para todo $x \in M$.

c) $\Phi_{k}(x, y)+\Phi_{k}(y, x) \geq 0$, para todo $x, y \in M$

d) $\Phi_{k}(x, y) \in \mathbb{R}$, para todo $x, y \in M$.

e) o potencial da ação $\Phi_{k}$ é Lipschitz.

3. Para $k>c(L), \Phi_{k}(x, y)+\Phi_{k}(y, x)>0$ se $x \neq y$.

Lema 1.3.7. A; função $C^{\infty}(M, \mathbb{R}) \ni \psi \mapsto c(L+\psi)$ é contínua na topologia da norma do supremo.

Definição 1.3.8. Dada uma medida boreliana de probabilidade $\mu \in T M$, definimos sua ação por:

$$
A_{L}(\mu)=\int_{T M} L d \mu
$$

Um teorema de Mañé [29], que relaciona o valor crítico com a ação das medidas é o seguinte:

Teorema 1.3.9. Se $M$ é compacta, então

$$
c(L)=-\min \left\{A_{L}(\mu): \mu \in M_{L}\right\}
$$

Além disso ao procurarmos curvas minimizantes elas estarão contidas no suporte de medidas minimizantes para a ação das medidas. No próximo capítulo veremos a proximidade entre estas medidas e a otimização ergódica. 



\section{CAPÍTULO 2}

\section{OTIMIZAÇÃO ERGÓDICA}

No capítulo anterior apresentamos dois problemas semelhantes, um proveniente do formalismo termodinâmico que consiste em estudar estados de equilíbrio e o outro da dinâmica Lagrangiana, que consiste em procurar medidas que minimizam a ação. Em otimização ergódica estamos interessados em estudar o problema variacional definido pelo funcional $A_{\phi}: \mathcal{M}_{f} \rightarrow \mathbb{R}$

$$
A_{\phi}(\mu)=\int \phi d \mu
$$

onde $\mathcal{M}_{f}$ ou $\mathcal{M}_{f}(X)$ denota o espaço das medidas borelianas de probabilidade $f$-invariantes, as funções $\phi: X \rightarrow \mathbb{R}$ e $f: X \rightarrow X$ são contínuas e $X$ é um espaço métrico compacto. Existem casos mais gerais onde $\phi$ não é contínua e $X$ não é compacto, porém outras condições são impostas para que o problema (2.1) fique bem definido.

Definição 2.0.10. Uma medida $\mu \in \mathcal{M}_{f}$ que maximiza o funcional $A_{\phi}$ é chamada $\phi$-maximizante, e o espaço das funções $\phi$-maximizantes será denotado por $\mathcal{M}_{\phi-\max }(f)$ ou $\mathcal{M}_{\max }(\phi)$.

Associado ao funcional (2.1) temos a soma de Birkhoff $S_{n} f=\sum_{i=0}^{n-1} \phi \circ f^{i}$, onde 
procuramos o máximo das médias temporais, isto é,

$$
\max _{x \in X}\left\{\lim _{n \rightarrow \infty} \frac{1}{n} S_{n} f(x)\right\}
$$

Os pontos $x$ tais que o limite em (2.2) atingem o supremo, bem como suas órbitas serão chamados de maximizantes.

Em geral, o limite $\lim _{n \rightarrow \infty} \frac{1}{n} S_{n} f(x)$ não precisa existir. Para contornar esse problema podemos usar dois caminhos. O primeiro é definirmos

$$
\operatorname{Reg}(X, f, \phi):=\left\{x \in X: \lim _{n \rightarrow \infty} \frac{1}{n} S_{n} f(x) \text { existe }\right\},
$$

e então calcularmos o supremo sobre esses pontos.

O segundo caminho é usar o limite superior no lugar do limite e, então, calcular o supremo sobre todos os pontos $x \in X$.

A partir disso podemos definir,

Definição 2.0.11. Seja $X$ um espaço topológico, e suponha que $\phi: X \rightarrow \mathbb{R}$ é contínua. Então:

$$
\begin{aligned}
\alpha(\phi) & =\alpha(\phi, f)=\sup _{m \in \mathcal{M}_{f}} \int_{X} \phi d m \\
\beta(\phi) & =\beta(\phi, f)=\sup _{x \in \operatorname{Reg}(X, f, \phi)} \lim _{n \rightarrow \infty} \frac{1}{n} S_{n} f(x) \\
\gamma(\phi) & =\gamma(\phi, f)=\operatorname{suplimsup}_{x \in X} \frac{1}{n \rightarrow \infty} S_{n} f(x) \\
\delta(\phi) & =\delta(\phi, f)=\limsup _{n \rightarrow \infty} \frac{1}{n} \sup _{x \in X} S_{n} f(x) .
\end{aligned}
$$

Em [13] Conze e Guivarc'h mostraram que se $f$ for contínua e $X$ for compacto então esses valores são iguais. Este resultado fornece mais ferramentas para atacar o problema (2.1), assim como o seguinte teorema:

Teorema 2.0.12 (Teorema de Dualidade de Fenchel-Rockafellar [36]). Seja $Z$ um espaço vetorial topológico localmente convexo sobre $\mathbb{R}$, com dual $Z^{*}$. Suponha 
$H: Z \rightarrow(-\infty,+\infty]$ convexa $^{1}$ e $G: Z \rightarrow[-\infty,+\infty)$ côncava ${ }^{2}$. Então, se existe um ponto $z_{0} \in Z$ onde ambas $H$ e $G$ são finitas e ao menos uma delas é contínua, vale que:

$$
\min _{x \in Z^{*}}\left\{H^{*}(x)-G^{*}(x)\right\}=\sup _{z \in Z}\{G(z)-H(z)\}
$$

onde $H^{*}(x)=\sup _{z \in Z}\{\langle z, x\rangle-H(z)\} e G^{*}(x)=\sup _{z \in Z}\{\langle z, x\rangle-G(z)\}$ e $\langle z, x\rangle$ indica a paridade dual entre $Z$ e $Z^{*}$.

Através deste teorema é possível mostrar ([15]) para o shift unilateral $\sigma$ sobre um alfabeto de $d$ símbolos $\Sigma$, que dado um potencial contínuo $\phi$ vale:

$$
\alpha(\phi)=\inf _{f \in C^{0}(\Sigma)} \sup _{x \in \Sigma}\{\phi(x)+f \circ \sigma(x)-f(x)\} .
$$

Observação 2.0.13. Esta igualdade dual vale em contextos mais gerais. Em [13], os autores à demonstraram para uma aplicação contínua qualquer $f: X \rightarrow X$ definida sobre um espaço métrico compacto $X$.

Uma função $f$ que cumpre o ínfimo é de particular interesse:

Definição 2.0.14. Uma função $u \in C^{0}(X)$ é chamada uma sub-ação para o potencial $\phi$, quando vale sobre $X$ a seguinte expressão:

$$
\phi+u \circ f-u \leq \alpha(\phi) .
$$

Estas funções foram estudadas em diversos trabalhos [3] [10] [16] [17] [25] [26], e são importantes para fornecer informações sobre as medidas $\phi$-maximizantes, pois quando existe uma sub-ação $u$ para $\phi$, teremos que o suporte de cada medida maximizante estará contido na pré-imagem de $\alpha(\phi)$ pela função $B=\phi+u \circ f-u$.

\footnotetext{
${ }^{1}$ Uma função $f: Z \rightarrow \mathbb{R}$ é chamada convexa se para qualquer $x, y \in Z$ temos $f(x t+(t-1) y) \leq$ $t f(x)+(1-t) f(y)$, onde $t \in[0,1]$.

${ }^{2}$ Uma função $f: Z \rightarrow \mathbb{R}$ é chamada côncava se para qualquer $x, y \in Z$ temos $f(x t+(t-1) y) \geq$ $t f(x)+(1-t) f(y)$, onde $t \in[0,1]$.
} 


\subsection{O conjunto das medidas maximizantes}

O objeto de interesse da otimização ergódica é estudar a relação entre essas medidas maximizantes, seus suportes e as funções $\phi$ e $f$. Daí, segue a importância de estudar a existência dessas medidas e a caracterização de $\mathcal{M}_{\max }(\phi)$.

Teorema 2.1.1. Se $X$ é um espaço métrico compacto e $\phi: X \rightarrow \mathbb{R}$ é contínua, então $\mathcal{M}_{\text {max }}(\phi) \neq \emptyset$.

Abaixo, segue a generalização:

Teorema 2.1.2. Seja $f: X \rightarrow X$ uma aplicação contínua num espaço métrico compacto e suponha que $\phi: X \rightarrow \mathbb{R}$ é semi-contínua superiormente. Então

i) $\mathcal{M}_{\max }(\phi) \neq \emptyset$.

ii) $\mathcal{M}_{\text {max }}(\phi)$ é convexo e compacto.

iii) os pontos extremais de $\mathcal{M}_{\text {max }}(\phi)$ são precisamente aquelas medidas maximizantes que são ergódicas. Em particular, existe ao menos uma medida $\phi$-maximizante ergódica.

Com respeito a caracterização do conjunto das medidas maximizantes, temos o seguinte resultado devido a O. Jenkinson [21]:

Teorema 2.1.3. Dado $A \subset \mathcal{M}_{f}$, são equivalentes:

1) Existe $\phi \in C(X)$ tal que $A=\mathcal{M}_{\max }(\phi)$

2) A é uma face ${ }^{1}$ de $\mathcal{M}_{f}$

Como consequência deste teorema temos três resultados, todos com a hipótese de $X$ ser um espaço métrico compacto.

\footnotetext{
${ }^{1}$ Um subconjunto convexo e não-vazio $F \subset K$, com $K$ convexo, é chamado de face de $K$ se, quando $\lambda k_{1}+(1-\lambda) k_{2} \in F$ com $k_{1}, k_{2} \in K$ e $0<\lambda<1$, então $k_{1}, k_{2} \in F$.
} 
Teorema 2.1.4. Suponha que o conjunto das medidas ergódicas de $\mathcal{M}_{f}$, denotado por $\mathcal{M}_{\text {erg }}$, é um subconjunto fechado de $\mathcal{M}_{f}$ na topologia fraca $*$. Para todo subconjunto não vazio $\mathcal{E} \subset \mathcal{M}_{\text {erg }}$, existe uma função contínua $\phi: X \rightarrow \mathbb{R}$, tal que, $\mathcal{M}_{\max }(\phi)$ é o fecho da envoltória convexa de $\mathcal{E}$.

Teorema 2.1.5. Seja $\mathcal{E} \neq \emptyset$ um subconjunto de $\mathcal{M}_{\text {erg }}$ que é fechado (fraco *) em $\mathcal{M}_{f}$. Então existe uma função contínua $\phi: X \rightarrow \mathbb{R}$, tal que, $\mathcal{M}_{\max }(\phi)$ é o fecho da envoltória convexa de $\mathcal{E}$.

Teorema 2.1.6. Seja $\mu \in \mathcal{M}_{\text {erg }}$, então existe uma função contínua $\phi: X \rightarrow \mathbb{R}$, tal que $\mu$ é a única medida $\phi$-maximizante.

Em outro trabalho O. Jenkinson [23], considerou a dinâmica $2 x$ no intervalo e definiu uma ordem parcial sobre $\mathcal{M}_{2 x}$. Uma medida $\mu$ é majorada por $\nu$ e escrevemos $\mu \prec \nu$ se para toda função convexa $\phi:[0,1] \rightarrow \mathbb{R}$, tivermos:

$$
\mu(\phi)=\int \phi d \mu \leq \int \phi d \nu=\nu(\phi) .
$$

Com essa ordem parcial temos que as medidas maximais de $\left(\mathcal{M}_{2 x}, \prec\right)$ são os deltas de diracs $\delta_{0}$ e $\delta_{1}$, porém a existência de medidas minimais não é imediata. Definindo $\mathcal{M}_{q}=\left\{\mu \in \mathcal{M}_{2 x}: \int x d \mu(x)=q\right\}$ o autor provou o seguinte teorema:

Teorema 2.1.7. Para todo $q \in[0,1]$ o conjunto ordenado $\left(\mathcal{M}_{q}, \prec\right)$ possui um elemento minimal, este elemento é uma medida de Sturmian ${ }^{1}$.

\subsection{Alguns dos Principais Resultados Conhecidos}

As motivações de se maximizar ou minimizar o funcional (2.1) vem primeiramente do formalismo termodinâmico e dos sistemas Lagrangianos. No primeiro caso podemos considerar o potencial $\frac{1}{T} \phi: X \rightarrow \mathbb{R}$, onde $T$ representa a temperatura associada a um sistema físico. A pressão para este potencial será:

\footnotetext{
${ }^{1}$ Uma medida de $\mathcal{M}_{f}$ tal que seu suporte está contido em um semi-círculo fechado.
} 


$$
P(\phi)=\sup _{\mu \in \mathcal{M}_{f}}\left\{h_{\mu}(f)+\frac{1}{T} \int \phi d \mu\right\}
$$

desta forma estudar o problema (2.1) se resume a procurar estados de equilíbrio quando a temperatura é zero. Quando a temperatura $T$ vai a zero, o primeiro termo na expressão da pressão, que é a entropia métrica, torna-se desprezível. Assim, o segundo termo será o principal responsável pelo valor de $P(\phi)$. Alguns trabalhos na área de otimização ergódica podem ser encontrados estudando esta relação, por exemplo em [22], os autores analisaram o comportamento dos estados de equilíbrios de Gibbs $\mu_{T}$ (olhar [22] para definição). Quando a temperatura $T$ vai a zero, eles mostraram que qualquer ponto de acumulação (no sentido fraco*) será uma medida maximizante, além disso, apenas as probabilidades que maximizam a entropia podem ser pontos de acumulação. Eles também estudaram a relação entre a convergência dos estados de equilíbios e as medidas $\phi$-maximizantes. Morris [ ] e Kempton [ ] deram continuidade ao estudo feito em [22].

Outra motivação se origina dos sistemas Lagrangianos, e a principal diferença está no fato de que, quando procuramos medidas minimizantes para a ação das medidas, a função considerada dentro da integral é o Lagrangiano, que está conectado com a dinâmica através da equação de Euler-Lagrange. Já na otimização ergódica a função $\phi$ não precisa estar relacionada com a dinâmica $f$.

Uma das mais importantes conjecturas na área de otimização ergódica (2.2.2) é baseada numa conjectura para sistemas Lagrangianos devido a R. Mañé [29]:

Conjectura 2.2.1. Para um Lagrangiano genérico ${ }^{1}$, exisite uma única medida minimizante suportada em uma órbita periódica.

Conjectura 2.2.2. Seja $f: X \rightarrow X$ transitiva, e hiperbólica com uma estrutura local de produto ${ }^{2}$, onde $X$ é um espaço métrico compacto. Então genericamente para

\footnotetext{
${ }^{1}$ Genérico no sentido de Mañé: Uma propriedade $P$ é dita genérica para o lagrangiano $L$ se existe um conjunto residual $\mathcal{O}$ em $C^{\infty}(M, \mathbb{R})$, tal que, se $\psi \in \mathcal{O}$, então $L+\psi$ tem a propriedade $P$.

${ }^{2}$ Seja $W_{\epsilon}^{s}(x)=\left\{y \in X: d\left(f^{n} x, f^{n} y\right) \leq \epsilon\right.$ para todo $\left.n \geq 0\right\}$ e $W_{\epsilon}^{u}(x)=\left\{y \in X: d\left(f^{-n} x, f^{-n} y\right) \leq\right.$
} 
$\phi: X \rightarrow \mathbb{R}$ Lipschitz existe uma medida $\phi$-maximizante suportada em órbita periódica.

Apesar da conjectura (2.2.1) ter sido respondida de maneira negativa em alguns casos, como por exemplo, por D. Massart [30], que construiu um Lagrangiano L no 2-toro que não satisfaz a generecidade das medidas periódicas na topologia $C^{4}$, a conjectura (2.2.2) continua em aberto e já foi provada em certos casos como por exemplo em [35], para o espaço das funções chamadas $A$-supercontínuas.

Definição 2.2.3. Seja $A_{n} \searrow 0$ com a propriedade que $\frac{A_{n}}{A_{n+1}} \rightarrow \infty$. Uma função $\phi: X \rightarrow \mathbb{R}$ é chamada A-supercontínua se existe $C>0$ tal que,

$$
|f(x)-f(y)| \leq C A_{n} \text { sempre que } d(x, y) \leq 2^{-n} .
$$

O espaço das funções $A$-supercontínuas é denotado por $C_{A}(X)$ e com a norma natural é um espaço de Banach. A. Quas e J. Siefken, demonstraram o seguinte teorema: Teorema 2.2.4. Seja $P=\left\{\phi \in C_{A}(X): \exists\right.$ ! medida periódica que maximiza $\left.\int \phi d \mu\right\}$. Então, $P$ contém um subconjunto aberto e denso em $C_{A}(X)$, sobre a topologia da $A$ norma.

Nesta direção Lopes, Contreras e Thieullen demonstraram o seguinte teorema:

Teorema 2.2.5 ([27]). Seja $f$ a aplicação $2 x$ em $S^{1}$. Seja $H_{\alpha}$ o conjunto das funções $\alpha$-Hölder em $S^{1}$ e seja $\mathcal{F}_{\alpha+}=\bigcup_{\beta>\alpha} H_{\beta}$. Seja $\mathcal{P}_{\alpha+} \subset \mathcal{F}_{\alpha+}$ o subconjunto de funções unicamente maximizadas por medidas periódicas. Então $\mathcal{P}_{\alpha+}$ contém um conjunto aberto que é denso em $\mathcal{F}_{\alpha+}$ na topologia de $H_{\alpha}$.

Outro resultado nesta linha é devido a Yang et. al.:

Teorema 2.2.6 ([40]). Seja $f$ o shift unilateral ou bilateral e seja $C_{\text {Lip }}$ a classe das funções Lipschitz. Para qualquer $\phi \in C_{\text {Lip }}$ maximizada por uma medida gerada por um ponto não periódico, existe uma pertubação de $\phi$ tal que aquela medida não é mais $\phi$-maximizante.

$\epsilon$ para todo $n \geq 0\}$ dizemos que $f$ tem uma estrutura local de produto se existem $\delta, \epsilon>0$ tal que se $x, y \in X \operatorname{com} d(x, y)<\delta$ então $W_{\epsilon}^{s}(x) \cap W_{\epsilon}^{u}(y)$ é um único ponto. 
Um resultado análogo à conjectura (2.2.2) foi obtido por Bousch.

Definição 2.2.7. Uma função $\phi$ é uma função de Walters se para cada $\epsilon>0$ existe um $\delta>0$ tal que, para todo $n \in \mathbb{N}$ e $x$ e $y$,

$$
\max _{0 \leq i<n}\left\{d\left(f^{i} x, f^{i} y\right)\right\} \leq \delta \Rightarrow\left|S_{n} f(x)-S_{n} f(y)\right|<\epsilon,
$$

onde $S_{n} f(x)=\sum_{i=0}^{n-1} \phi\left(f^{i}(x)\right)$.

Teorema 2.2.8 ( [8] ). Seja $f$ a aplicação $2 x$ em $S^{1}$ e seja $W$ o conjunto das funções de Walters. Se $P \subset W$ é o conjunto das funções de Walters maximizadas por medidas periódicas, então $P$ contém um conjunto aberto e denso em $W$ na topologia da norma Walters.

Uma observação importante é de que as funções super-contínuas são Lipschitz e as funções Lipschitz são funções de Walters, mas a conjectura (2.2.2) não está respondida para o caso das funções Lipschitz.

Outros autores consideraram o estudo de medidas maximizantes com suportes diferentes de órbitas periódicas. No trabalho de O. Jenkinson [18], ele mostrou que admitindo certa regularidade da dinâmica temos que, ter medida maximizante sem suporte total é uma propriedade genérica em $C^{r, \alpha}$. Mais especificamente ele mostrou:

Teorema 2.2.9. Seja $f: X \rightarrow X$ transitiva, hiperbólica e com uma estrutura local de produto. Suponha que X não consiste de uma órbita periódica, então

$$
P=\left\{\phi \in C^{r, \alpha}(X): \text { toda medida } \phi \text {-maximizante não tem suporte total }\right\}
$$

é aberto e denso em $C^{r, \alpha}$.

Teorema 2.2.10. Seja $f: X \rightarrow X$ transitiva, hiperbólica e com uma estrutura local de produto. Então uma função genérica em $C^{0}$ tem uma única medida maximizante, e esta medida é periódica.

Apesar destes resultados ainda existe alguns problemas em aberto sobre medidas maximizantes com suportes totais, podemos citar o seguinte: 
Problema: Seja $f: X \rightarrow X$ transitiva, hiperbólica e com uma estrutura local de produto. Encontre um exemplo explícito de uma função contínua com uma única medida maximizante de suporte total.

Os trabalhos referenciados até aqui consideraram a dinâmica fixa e a função de dentro da integral variando sobre algum espaço adequado. Consideraremos agora o contrário, vamos fixar a função $\phi$ e variar a dinâmica, nestas condições temos os seguintes teoremas retirados de [41] [42] [1].

Teorema 2.2.11. Seja End $\left(S^{1}\right)$ o conjunto de endomorfismos do círculo, então o conjunto das funções $f \in \operatorname{End}\left(S^{1}\right)$ tais que existe uma medida de probabilidade boreliana f-invariante $\phi$-maximizante suportada em uma órbita periódica é denso no conjunto dos endomorfismos na topologia $C^{0}$.

Teorema 2.2.12. Seja Hom $(X)$ o espaço dos homeomorfismos de uma variedade Riemanianna compacta $X$, então o conjunto das $f \in \operatorname{Hom}(X)$ tais que existe uma medida de probabilidade boreliana $f$-invariante $\phi$-maximizante suportada em uma órbita periódica é denso no conjunto dos homeomorfismos na topologia $C^{0}$.

Teorema 2.2.13. Dada uma função localmente não constante $g: X \rightarrow \mathbb{R}$ definida em uma variedade Riemanniana compacta conexa $X$ de dimensão maior ou igual a dois, então o complementar de $\operatorname{Hom}_{\operatorname{Per}(\phi)}(X)$ é residual. Onde $\operatorname{Hom}_{P e r(\phi)}(X)$ denota o conjunto dos homeomorfismos $f: X \rightarrow X$, que tem medida $\phi$-maximizante $f$ invariante suportada em órbita periódica.

Nosso trabalho é uma generalização do primeiro teorema, pois consideramos o conjunto de endomorfismos sobre uma variedade compacta. O segundo teorema é um resultado similar considerando homeomorfismos e o terceiro mostra que não poderemos ter generecidade. 



\section{CAPÍTULO 3}

\subsection{Definição do problema}

Primeiramente vamos fixar a notação que será usada. $M$ é uma variedade Riemanniana $n$-dimensional, compacta, conexa e sem bordo, $f \in \operatorname{End}(M)$, isto é, $f: M \rightarrow M$ contínua e sobrejetora, e $\phi: M \rightarrow \mathbb{R}$ é uma função contínua. Denotemos por $\mathcal{M}_{f}$ o conjunto das medidas borelianas de probabilidades $f$-invariantes e $\mu_{\max } \in \mathcal{M}_{f}$ uma medida $\phi$-maximizante, i.e., $\sup _{\nu \in \mathcal{M}_{f}} \int \phi d \nu=\int \phi d \mu_{\max }$. Sem perda de generalidade podemos supor que $\int \phi d \mu_{\max }=0$, caso contrário subtraímos uma constante de $\phi$. Além disso, suponhamos que esta medida é ergódica, pois estamos maximizando um funcional linear sobre o convexo $\mathcal{M}_{f}$ onde as medidas ergódicas são os pontos extremais, e também consideraremos que $\mu_{\max }$ não está suportada em uma órbita periódica, pois senão já teríamos o resultado que buscamos.

Como toda variedade pode ser triangularizada por simplexos arbitrariamente pequenos [33], podemos aproximar $f$ por um endomorfismo $g$ que é de classe $C^{1}$ em cada um destes simplexos, e que é tão próximo quanto se queira de $f$ na topologia $C^{0}$. Além disso, para cada simplexo, $D g$ pode ser tomada como sendo constante e de posto máximo em alguma das cartas que contém este simplexo. Isto implica que $g$ é injetora 
restrita a cada simplexo, em particular, se $k$ é o número de simplexos da triangulação de $M$, então cada ponto de $M$ possui, no máximo, $k$ pré-imagens por $g$.

Assim, o conjunto dos endomorfismos de $M$ para os quais, existe uma cota superior finita para a cardinalidade das pré-imagens de um ponto, é denso na topologia $C^{0}$. Iremos assumir desde o início que $f$ satisfaz esta propriedade. Além disso:

Proposição 3.1.1. Seja $f: M \rightarrow M$ um endomorfismo tal que, para cada $p \in M$, tem um número finito de pré-imagens pela $f$ e $h: M \rightarrow M$ um homeomorfismo, então $p$ terá um número finito de pré-imagens pela função $g=h \circ f$.

Demonstração. Se $h$ é homeomorfismo e $h^{-1}(p)=q$, então

$$
g^{-1}(p)=(h \circ f)^{-1}(p)=f^{-1} \circ h^{-1}(p)=f^{-1}(q) .
$$

Como para todo ponto de $M$ existe um número finito de pré-imagens pela $f$, segue o resultado.

Observação 3.1.2. O número de pré-imagens não é necessariamente igual para todo $p \in M$.

Nosso objetivo principal é provar o seguinte teorema:

Teorema 3.1.3. Seja $M$ uma variedade Riemanniana compacta, conexa e sem bordo. Dados um endomorfismo $f: M \rightarrow M$, uma função contínua $\phi: M \rightarrow \mathbb{R} e \varepsilon>0$, existe um endomorfismo $\tilde{f}: M \rightarrow M$ tal que

$$
d(f, \tilde{f})=\max _{x \in M} d(f(x), \tilde{f}(x))<\varepsilon,
$$

e existe uma medida $\phi$-maximizante para $\tilde{f}$ suportada em uma órbita periódica.

Observação 3.1.4. A medida periódica encontrada não é necessáriamente a única medida $\phi$-maximizante para $\tilde{f}$.

Antes de começar a construção da função $\tilde{f}$ apresentaremos alguns lemas importantes. O primeiro, retirado de [41], é uma forma particular do resultado conhecido como lema de Atkinson [2]: 
Lema 3.1.5. Seja $A \subset \operatorname{supp}\left(\mu_{\max }\right)$ um conjunto de pontos não periódicos com $f(A) \subset$ $A$ e $\mu_{\max }(A)=1$. Suponha que existe $\varepsilon^{*}>0$ tal que para todo ponto $x \in A$ e $n>0$ com $d\left(f^{n}(x), x\right)<\varepsilon^{*}, S_{n} f(x)=\sum_{i=0}^{n-1} \phi\left(f^{i}(x)\right) \leq 0$. Então, para todo $x \in A$ e $n>0$ $\operatorname{com} d\left(f^{n}(x), x\right)<\frac{\varepsilon^{*}}{2}$ teremos $S_{n} f(x)=0$.

Demonstração. Suponhamos por absurdo que existe $x \in A$ e $n>0$ tal que $d\left(f^{n}(x), x\right)<\frac{\varepsilon^{*}}{2}$ e $S_{n} f(x)=-\delta<0$. Como $x$ não é ponto periódico podemos escolher $K>8$ inteiro, tal que, $\min _{1 \leq i \leq n} d\left(f^{i}(x), x\right)>\frac{2 \varepsilon^{*}}{K}$. Como $A \subset \operatorname{supp}\left(\mu_{\max }\right)$, existe $\tilde{A} \subset A$ de medida $\mu_{\max }$ positiva, com $\tilde{A} \subset B\left(x ; \frac{\varepsilon^{*}}{K}\right)$ tal que, para todo $y \in \tilde{A}$ temos

$$
d\left(f^{n}(y), x\right) \leq d\left(f^{n}(y), f^{n}(x)\right)+d\left(f^{n}(x), x\right)<\frac{\varepsilon^{*}}{M}+\frac{\varepsilon^{*}}{2},
$$

para algum $M>0$ e

$$
S_{n} f(y)<\frac{-\delta}{2},
$$

isto é possível pela continuidade das funções $f$ e $\phi$.

Como $\mu_{\max }(\tilde{A})>c>0$ para algum $c$ real, temos pelo teorema de recorrência de Poicaré que $\mu_{\text {max }}$-qtp $x \in \tilde{A}$ é recorrente. Assim, para cada $y \in \tilde{A}$, se o diâmetro de $\tilde{A}$ for suficientemente pequeno, $N(y)>n$ é o primeiro retorno de $y$ a $\tilde{A}$.

Afirmação: Existe $y \in \tilde{A}$ tal que

$$
S_{N(y)} f(y) \geq-\frac{\delta}{4}
$$

Suponha que para todo $y \in \tilde{A}, S_{N(y)} f(y)<-\frac{\delta}{4}<0$. Pelo teorema de Birkhoff (lembrando que $\mu_{\max }$ é ergódica) temos que para $\mu_{\max }$-qtp $y \in \tilde{A}$,

$$
\frac{1}{N} \sum_{i=0}^{N-1} \chi_{\tilde{A}} \circ f^{i}(y) \approx \int \chi_{\tilde{A}} d \mu_{\max }=\mu_{\max }(\tilde{A})>c
$$

desta forma tiramos

$$
\#\left\{i \in\{0,1,2, \ldots, N-1\}: f^{i}(y) \in \tilde{A}\right\}>c N
$$


com $N$ suficientemente grande.

Para $y \in \tilde{A}$ recorrente, denotemos por $N_{k} \rightarrow \infty$ os tempos de retornos, temos,

$$
S_{N_{k}} f(y)=S_{N_{1}} f(y)+S_{N_{2}-N_{1}} f^{N_{1}}(y)+\ldots+S_{N_{k}-N_{k-1}} f^{N_{k-1}}(y)<-\frac{\delta}{4} k<-c \frac{\delta}{4} N_{k},
$$

resultando

$$
\lim _{k \rightarrow \infty} \frac{1}{N_{k}} S_{N_{k}} f(y)<-c \frac{\delta}{4} .
$$

Isto é um absurdo, pois encontramos um conjunto de medida positiva onde não vale o teorema ergódico de Birkhoff, i.e., $\mu_{\max }$-qtp $x \in M$

$$
\lim _{n \rightarrow \infty} \frac{1}{n} S_{n} f(x)=\int \phi d \mu_{\max }=0 .
$$

Assim chegamos que existe um $y \in \tilde{A} \operatorname{com} N(y)>n$ tal que:

$$
S_{N(y)} f(y) \geq-\frac{\delta}{4} \quad \text { e } \quad S_{n} f(y)<-\frac{\delta}{2} .
$$

Destas expressões e da desigualdade (3.1) tiramos que existe $z=f^{n}(y) \in A$ com

$$
\begin{aligned}
d\left(f^{N(y)-n}(z), z\right) & =d\left(f^{N(y)}(y), f^{n}(y)\right) \\
& \leq d\left(f^{N(y)}(y), x\right)+d\left(f^{n}(y), x\right) \\
& \leq \frac{\varepsilon^{*}}{K}+\frac{\varepsilon^{*}}{M}+\frac{\varepsilon^{*}}{2}<\varepsilon^{*}
\end{aligned}
$$

onde, $d\left(f^{N(y)}(y), x\right)<\frac{\varepsilon^{*}}{K}$ pois $f^{N(y)}(y) \in B\left(x ; \frac{\varepsilon^{*}}{K}\right)$. Além disso,

$$
S_{N(y)-n} f(z)=S_{N(y)} f(y)-S_{n} f(y)>\frac{\delta}{2} .
$$

Mas isto é uma contradição pois, por hipótese $S_{N(y)-n} f(z) \leq 0$.

A próxima proposição segue como corolário dos resultados de O. Jenkinson [8], e é uma consequência da compacidade fraca-* de $\mathcal{M}_{f}$.

Proposição 3.1.6. Se $\int \phi d \mu_{\max }=0$, temos que para todo $a>0$ existe um $m_{0}(a)>0$ tal que para todo $m>m_{0}(a)$

$$
\delta(\phi)=\frac{1}{m} S_{m} f(x)<\frac{a}{2}, \text { para todo } x \in M .
$$


Demonstração. Segue do fato de que

$$
\limsup _{n \rightarrow \infty} \max _{x \in M} \frac{1}{n} S_{n} f(x)=\sup _{\mu \in \mathcal{M}_{f}} \int \phi d \mu=0 .
$$

Agora denotemos por $C$ o conjunto dos pontos em $\operatorname{supp}\left(\mu_{\max }\right)$ que são recorrentes, este conjunto é invariante pela $f$ e pelo teorema de Poicaré este conjunto tem medida total.

Sejam $\bar{x} \in C$ e $y=f^{n}(\bar{x}) \in C$ tais que $d(\bar{x}, y)<\varepsilon_{1}=\min \left\{\frac{\varepsilon^{*}}{8}, \varepsilon\right\}$, onde $\varepsilon^{*}$ vem do lema 3.1.5 e $\varepsilon$ do teorema 3.1.3. Vamos renomear $\varepsilon_{1}=\varepsilon$ e denotemos por $B(\bar{x} ; \varepsilon)$ a bola aberta com centro em $\bar{x}$ e raio igual a $\varepsilon$, e por $B[\bar{x} ; \varepsilon]$ a bola fechada. Podemos escolher $\bar{x}$ e $y$ de maneira que, para todo inteiro $0<i<n, f^{i}(\bar{x}) \notin B(\bar{x} ; \varepsilon)$.

Temos duas possibilidades:

Caso 1) Para todo $\bar{x} \in C$ e $n>0$ temos que, se $d(\bar{x}, y)<\varepsilon$, então $S_{n} f(\bar{x}) \leq 0$;

Caso 2) Existe pelo menos um $x_{0} \in C$ e $n_{0}>0$ tal que $d\left(x_{0}, f^{n_{0}}\left(x_{0}\right)\right)<\varepsilon$ e, tal que $S_{n_{0}} f\left(x_{0}\right)>0$.

Antes de analisarmos estes casos, apresentamos a seguinte proposição:

Proposição 3.1.7. Sejam $f: M \rightarrow M$ um endomorfismo e $\phi: M \rightarrow \mathbb{R}$ uma função contínua. Se para algum $x$ periódico de período $n>0$ vale que, para todo $y \in M$

$$
\limsup _{k \rightarrow \infty} \frac{1}{k} S_{k} f(y) \leq \frac{1}{n} S_{n} f(x)
$$

então a medida suportada na órbita de $x$ é $\phi$-maximizante.

Demonstração. Seja $\mu_{\max }$ uma medida $\phi$-maximizante ergódica. Temos que

$$
\sup _{\mu \in \mathcal{M}_{f}} \int \phi d \mu=\int \phi d \mu_{\max }=\limsup _{k \rightarrow \infty} \frac{1}{k} S_{k} f(y) \leq \frac{1}{n} S_{n} f(x)=\int \phi d \mu_{x}
$$

onde $\mu_{x}=\frac{1}{n} \sum_{i=0}^{n-1} \delta_{f^{i}(x)}$ é a medida suportada na órbita periódica de $x$. 


\subsection{Caso 1}

Consideramos que estamos sob as hipóteses do caso 1 e vamos subdividir este caso em outros dois:

1.1. Para todo $x \in B[\bar{x} ; \varepsilon]$, se $f^{n}(x) \in B[\bar{x} ; \varepsilon]$ temos $S_{n} f(x) \leq 0$;

1.2. Existe um $x_{0} \in B[\bar{x} ; \varepsilon]$ e $n_{0} \operatorname{com} f^{n_{0}}\left(x_{0}\right) \in B[\bar{x} ; \varepsilon]$ e $S_{n_{0}} f\left(x_{0}\right)>0$.

\subsubsection{Caso 1.1.}

Como $y=f^{n}(\bar{x}) \in C$, existe um $k>0$ inteiro que é o tempo de primeiro retorno de $y$ a $B(\bar{x} ; \varepsilon)$. Assim, construímos uma função $T: M \rightarrow M$ definida como sendo a identidade fora da $B[\bar{x} ; \varepsilon]$ e constante igual a $\bar{x}$ para todo $x \in B\left(f^{k}(y) ; \delta\right)$, onde delta é pequeno o suficiente para que a vizinhança de $f^{k}(y)$ esteja inteiramente contida dentro de $B(\bar{x} ; \varepsilon)$ e para que $y \notin B\left(f^{k}(y) ; \delta\right)$. Podemos escolher $T$ de maneira que seja contínua em $M$ e que $\tilde{f}=T \circ f$ seja sobrejetora. Além disso, $\bar{x}$ será um ponto periódico para $\tilde{f}$ com período $n+k$ e, pelo lema 3.1.5, $S_{n+k} f(\bar{x})=0$.

Para provar que a medida suportada na órbita periódica criada é $\phi$-maximizante note que, fora de $B(\bar{x} ; \varepsilon)$ não alteramos a dinâmica, ou seja, basta nos preocuparmos com os pontos de $B(\bar{x} ; \varepsilon)$ que retornam a $B(\bar{x} ; \varepsilon)$. Desta forma, para $x \in B(\bar{x} ; \varepsilon)$, ou esse ponto retorna infinitas vezes por $\tilde{f}=T \circ f$ ao conjunto ou uma quantidade finita de vezes. Na segunda hipótese, existe um $i>0$ tal que $\tilde{f}^{m}(x) \notin B(\bar{x} ; \varepsilon)$ para todo $m>i$ assim

$$
\lim _{n \rightarrow \infty} \frac{1}{n} S_{n} \tilde{f}(x)=\lim _{k \rightarrow \infty} \frac{1}{k+m} S_{k} \tilde{f}\left(\tilde{f}^{m}(x)\right)=\lim _{k \rightarrow \infty} \frac{1}{k} S_{k} f\left(\tilde{f}^{m}(x)\right) \leq 0 .
$$

Agora se considerarmos que $x$ retorna infinitas vezes para $B(\bar{x} ; \varepsilon)$, digamos $N_{1}, N_{2}, \ldots$, teremos que

$$
\begin{aligned}
\frac{1}{N_{k}} S_{N_{k}} \tilde{f}(x) & =\frac{1}{N_{k}}\left[S_{N_{1}} \tilde{f}(x)+S_{N_{2}-N_{1}} \tilde{f}\left(\tilde{f}^{N_{1}}(x)\right)+\ldots+S_{N_{k}-N_{k-1}} \tilde{f}\left(\tilde{f}^{N_{k-1}}(x)\right)\right] \\
& =\frac{1}{N_{k}}\left[S_{N_{1}} f(x)+S_{N_{2}-N_{1}} f\left(\tilde{f}^{N_{1}}(x)\right)+\ldots+S_{N_{k}-N_{k-1}} f\left(\tilde{f}^{N_{k-1}}(x)\right)\right] \leq 0,
\end{aligned}
$$


assim, para todo $x$ :

$$
\limsup _{n \rightarrow \infty} \frac{1}{n} S_{n} \tilde{f}(x)=\lim _{k \rightarrow \infty} \frac{1}{N_{k}} S_{N_{k}} \tilde{f}(x) \leq 0 .
$$

Pela proposição (3.1.7) a medida suportada na órbita periódica é $\phi$-maximizante.

\subsubsection{Caso 1.2. e Caso 2}

Sejam $x_{0} \in B[\bar{x} ; \varepsilon]$ e $n_{0}>0$, tal que $f^{n_{0}}\left(x_{0}\right) \in B[\bar{x} ; \varepsilon]$ e $\frac{1}{n_{0}} S_{n_{0}} f\left(x_{0}\right)=a_{0}>0$. Pela proposição 3.1.6 tomemos $m_{0}=m\left(a_{0}\right)>n_{0}>0$. Agora para cada $k \in\left\{1,2, \ldots, m_{0}\right\}$ considere o compacto $K_{k}=B[\bar{x} ; \varepsilon] \cap f^{-k}(B[\bar{x} ; \varepsilon])$. Seja $q_{k}$ um elemento deste compacto que maximiza a função contínua $g_{k}(x)=\frac{1}{k} S_{k} f(x)$ definida em $K_{k}$, e tal que a distância $d\left(q_{k}, f^{n_{q_{k}}}\left(q_{k}\right)\right)$ é a menor possível, onde $n_{q_{k}}$ é o primeiro retorno de $q_{k}$ ao conjunto $B[\bar{x} ; \varepsilon]$.

Denotamos por $q$ um elemento que satisfaz:

$$
d\left(q, f^{n_{q}}(q)\right)=\min _{k \in\left\{1, \ldots, m_{0}\right\}}\left\{d\left(q_{k}, f^{n_{q_{k}}}\left(q_{k}\right)\right\}\right.
$$

Este elemento da forma que foi escolhido tem média máxima, i.e., para todo $x \in B[\bar{x} ; \varepsilon]$ e $j>0 \operatorname{com} f^{j}(x) \in B[\bar{x} ; \varepsilon]$ :

$$
\frac{1}{j} S_{j} f(x) \leq \frac{1}{n_{q}} S_{n_{q}} f(q)
$$

Vamos chamar de $q_{0}$ o valor do primeiro retorno de $q$, assim, $q_{0}=f^{n_{q}}(q)$. Note que este retorno pode acontecer:

a) no interior do conjunto $B[\bar{x} ; \varepsilon]$

b) na fronteira do conjunto $B[\bar{x} ; \varepsilon]$

O item $b$ ) será resolvido na próxima seção. Para o item a) definimos $\tilde{f}=T \circ f$ onde $T(x)=x$ fora de $B[\bar{x} ; \varepsilon]$ e, escolhemos $\delta>0$ tal que $B\left(f^{n_{q}}(q) ; \delta\right) \subset B(\bar{x} ; \varepsilon)$ e

$$
T(x)=q, \quad \forall x \in B\left(f^{n_{q}}(q), \delta\right)
$$

Desta forma $q$ é um ponto periódico para $\tilde{f}$. 
Lema 3.2.1. Dado $x \in B[\bar{x} ; \varepsilon]$, se $x$ volta infinitas vezes para $B[\bar{x} ; \varepsilon]$ nos tempos $N_{1}, N_{2}, \ldots$, então para todo inteiro $k>0$,

$$
\frac{1}{N_{k}} S_{N_{k}} \tilde{f}(x) \leq \frac{1}{n_{q}} S_{n_{q}} f(q)
$$

Demonstração. Para cada $x$ satisfazendo as hipóteses vale:

$$
\frac{1}{N_{k}} S_{N_{k}} f(x) \leq \frac{1}{n_{q}} S_{n_{q}} f(q)
$$

Seja $N_{0}=0$, temos que

$$
\begin{aligned}
\frac{1}{N_{k}} S_{N_{k}} \tilde{f}(x) & =\frac{1}{N_{k}}\left(\sum_{i=0}^{N_{1}-1} \phi \circ \tilde{f}^{i}(x)+\ldots+\sum_{i=N_{k-1}}^{N_{k}-1} \phi \circ \tilde{f}^{i}(x)\right) \\
& =\frac{1}{N_{k}}\left(\frac{N_{1}-N_{0}}{N_{1}-N_{0}} S_{N_{1}-N_{0}} \tilde{f}(x)+\ldots+\frac{N_{k}-N_{k-1}}{N_{k}-N_{k-1}} S_{N_{k}-N_{k-1}} \tilde{f}\left(\tilde{f}^{\left.\left.N_{k-1}(x)\right)\right)}\right.\right. \\
& =\frac{1}{N_{k}}\left(\sum_{i=1}^{k}\left(N_{i}-N_{i-1}\right) \frac{1}{N_{i}-N_{i-1}} S_{N_{i}-N_{i-1}} f\left(\tilde{f}^{N_{i-1}}(x)\right)\right) \\
& \leq \frac{1}{N_{k}} \sum_{i=1}^{k}\left(N_{i}-N_{i-1}\right) \frac{1}{n_{q}} S_{n_{q}} f(q)=\frac{1}{n_{q}} S_{n_{q}} f(q) .
\end{aligned}
$$

Onde a igualdade (3.2) segue de $S_{k} f(x)=S_{k} \tilde{f}(x)$ se $\tilde{f}^{i}(x) \notin B[\bar{x} ; \varepsilon], 0 \leq i \leq k-1$.

Desta forma todo $x \in M$ :

$$
\limsup _{n \rightarrow \infty} \frac{1}{n} S_{n} \tilde{f}(x) \leq \frac{1}{n_{q}} S_{n_{q}} f(q)
$$

Pela proposição (3.1.7) temos que a medida suportada na órbita periódica de $q$ é $\phi$ maximizante.

Agora o que resta para concluir a demonstração do teorema 3.1.3 é mostrar que conseguimos fechar uma órbita periódica, de maneira contínua, no caso em que o elemento que tem média máxima retorna a primeira vez para $B[\bar{x} ; \varepsilon]$ na fronteira deste conjunto. 


\subsection{O Retorno na Fronteira}

Para fechar uma órbita periódica no caso em que o elemento de média máxima retorna na fronteira do conjunto, precisaremos ter controle sobre as médias ergódicas parciais numa vizinhança do retorno. Vamos considerar a bola fechada $B\left[x^{*} ; \varepsilon^{*}\right]$, cujo centro denotado por $x^{*}$ é o ponto médio do segmento $\overline{q q_{0}}$, de raio $\varepsilon^{*}=d\left(x^{*}, q\right)=d\left(x^{*}, q_{0}\right)$. Observe que $B\left[x^{*} ; \varepsilon^{*}\right] \subset B[\bar{x} ; \varepsilon]$ e, da forma como foram escolhidos $q$ e $q_{0}$, temos:

Proposição 3.3.1. Existe $\delta>0$ tal que, se $x$ está na componente conexa de $f^{-j}\left(B\left[q_{0} ; \delta\right]\right) \bigcap\left(B\left[q_{0} ; \delta\right] \cup B\left[x^{*} ; \varepsilon^{*}\right]\right)$ em que q não está, então

$$
\frac{1}{j} S_{j} f(x)<\frac{1}{n_{q}} S_{n_{q}} f(q)
$$

para todo $j \in \mathbb{N}^{*}$.

Demonstração. Note que as pré-imagens de $q_{0}$ até o iterado no máximo $m_{0}, P=$ $\bigcup_{i=1}^{m_{0}} f^{-i}\left(q_{0}\right)$, são finitas. Pela continuidade das funções $f$ e $\phi$, se $x \in P \cap B\left[x^{*}, \varepsilon^{*}\right]$, $x \neq q$ e $f^{i}(x)=q_{0}$, então existe $\delta_{1}(x)>0$ tal que se $d(x, y)<\delta_{1}(x)$ temos $\frac{1}{i} S_{i} f(y)<$ $\frac{1}{n_{q}} S_{n_{q}} f(q)$. Além disso, como $f$ tem finitas pré-imagens para cada $\delta_{1}(x)$, existe $\delta_{2}(x)>$ 0 tal que a componente conexa de $f^{-i}\left(B\left(q_{0} ; \delta_{2}(x)\right)\right)$ que contém $x$, está contida em $B\left(x ; \delta_{1}(x)\right)$. Finalmente existe $\delta_{3}>0$ tal que se $f^{-i}\left(B\left(q_{0} ; \delta_{3}\right)\right)$ intersecta $B\left[x^{*} ; \varepsilon^{*}\right]$ então existe $x \in P$ nesta componente. Tomando $\delta=\min _{x \in P}\left\{\delta_{1}(x), \delta_{2}(x), \delta_{3}\right\}$ temos o resultado.

Observação 3.3.2. Note que a partir desta seção, a condição de se trabalhar em um contexto diferenciável se faz útil.

Agora vamos mostrar que de todos os elementos de $B\left[x^{*} ; \varepsilon^{*}\right]$ o único que tem retorno máximo neste conjunto é o ponto $q$.

Proposição 3.3.3. Se $w \in B\left[x^{*} ; \varepsilon^{*}\right]$ e $f^{\tilde{n}}(w)$ é seu primeiro retorno a $B\left[x^{*} ; \varepsilon^{*}\right]$, então

$$
\frac{1}{\tilde{n}} S_{\tilde{n}} f(w) \leq \frac{1}{n_{q}} S_{n_{q}} f(q)
$$


Demonstração. Se o primeiro retorno de $w$ ao conjunto $B\left[x^{*} ; \varepsilon^{*}\right]$ for igual ao primeiro retorno de $w$ ao conjunto $B(\bar{x} ; \varepsilon), \tilde{n}=n_{w}$ e pela maneira que escolhemos $q$ temos

$$
\frac{1}{\tilde{n}} S_{\tilde{n}} f(w) \leq \frac{1}{n_{q}} S_{n_{q}} f(q)
$$

Agora se o primeiro retorno de $w$ ao conjunto $B\left[x^{*} ; \varepsilon^{*}\right]$ acontece depois do que seu retorno ao conjunto $B(\bar{x} ; \varepsilon)$, podemos escrever $\tilde{n}=n_{1}+\ldots+n_{k}$ onde

$$
f^{n_{i}}(w) \in B(\bar{x} ; \varepsilon) \backslash B\left[x^{*} ; \varepsilon^{*}\right], 1 \leq i \leq k-1 .
$$

Desta forma,

$$
\begin{aligned}
\frac{1}{\tilde{n}} S_{\tilde{n}} f(w) & \leq \frac{1}{\tilde{n}} \sum_{i=1}^{k}\left(n_{i}-n_{i-1}\right) \frac{1}{n_{i}-n_{i-1}} S_{n_{i}-n_{i-1}} f\left(f^{n_{i-1}}(w)\right) \\
& \leq \max _{i \in\{1, \ldots, k\}} \frac{1}{n_{i}-n_{i-1}} S_{n_{i}-n_{i-1}} f\left(f^{n_{i-1}}(w)\right) \leq \frac{1}{n_{q}} S_{n_{q}} f^{n_{q}}(q) .
\end{aligned}
$$

Denotemos por $I=B\left[x^{*}, \varepsilon^{*}\right] \cup B\left[q_{0} ; \delta\right]$. Iremos construir um novo endomorfismo $\tilde{f}=T \circ f$, onde $T$ é a identidade em $M \backslash I$, e tal que existe um ponto periódico de $\tilde{f}$ em $I$ e a média de $\phi$ sobre a medida suportada nesta órbita periódica é estritamente positiva. Para que exista uma medida $\phi$-invariante com média superior a desta, é necessário que, para algum $x \in M, \lim _{n \rightarrow \infty} \frac{1}{n} S_{n} \tilde{f}(x)>0$. Assim se para algum $x \in M$, $x$ não retornar a $I$, teremos que $f^{n}(x)=\tilde{f}^{n}(x)$ para todo $n>0$, e então

$$
\lim _{n \rightarrow \infty} \frac{1}{n} S_{n} \tilde{f}(x)=\lim _{n \rightarrow \infty} \frac{1}{n} S_{n} f(x) \leq 0 .
$$

Portanto estaremos interessados apenas no conjunto de pontos que retornam a $I$. Seja

$$
D=I \cap\left(\bigcup_{i=1}^{\infty} f^{-i}(I)\right) .
$$

Sobre este conjunto podemos definir as seguintes funções:

$$
\begin{gathered}
N_{r e t}(x)=\inf \left\{j \in \mathbb{N}^{*}: f^{j}(x) \in I\right\} \\
f_{2}(x)=f^{N_{r e t}(x)}(x)
\end{gathered}
$$




$$
\psi(x)=\frac{1}{N_{r e t}(x)} \sum_{i=0}^{N_{r e t}(x)-1} \phi\left(f^{i}(x)\right) .
$$

A proposição 3.3.1 nos garante que, se $x \in D$ e $\psi(x)>\psi(q)$, então $x$ pertence à componente conexa de $f_{2}^{-1}\left(B\left[q_{0} ; \delta\right]\right)$ que contém $q$. Denotaremos esse conjunto por $W_{0}$. Agora seja $x_{\max }$ o elemento de $\overline{W_{0}}$ que maximiza $\psi$. Se este ponto tiver retorno no interior de $I$, fechamos uma órbita periódica nele através de um homeomorfismo $T$ que leva seu retorno em $x_{\max }$. O retorno de $x_{\max }$ é um ponto interior ao conjunto $B\left[q_{0} ; \delta\right]$. $T$ é a identidade fora de $I$.

Caso isso não aconteça, isto é, caso $f_{2}\left(x_{\max }\right)$ pertença a $\partial I$, podemos tomar $\alpha \in W_{0}$ tal que,

$$
4 m_{0}\left(\psi\left(x_{\max }\right)-\psi(\alpha)\right) \leq|\psi(\alpha)-\psi(q)|
$$

e tal que o retorno de $\alpha$ esteja no interior de $B\left[q_{0} ; \delta\right]$.

Consideremos um homeomorfismo $T_{1}: M \rightarrow M$ que leva $f_{2}(\alpha)$ em $\alpha$, isto é, $T_{1}\left(f_{2}(\alpha)\right)=\alpha$. Se denotarmos por $\overline{\alpha f_{2}(\alpha)}$ o seguimento ligando os pontos $\alpha$ e $f_{2}(\alpha)$, podemos escolher $T_{1}$ de maneira que seja a identidade fora de $V\left(\overline{\alpha f_{2}(\alpha)}, \delta_{3}\right)$, onde

$$
V\left(\overline{\alpha f_{2}(\alpha)}, \delta_{3}\right)=\left\{x \in M: \min _{y \in \overline{\alpha f_{2}(\alpha)}} d(x, y)<\delta_{3}\right\},
$$

e $\delta_{3}>0$, que foi escolhido de maneira que a vizinhança esteja contida no conjunto $I$.

Na figura (3.1) a região sombreada denota a vizinhança do segmento $\overline{\alpha f_{2}(\alpha)}$.

Seja $\tilde{f}=T_{1} \circ f$. Note que, se $x \in D$ retorna infinitas vezes por $\tilde{f}$ ao conjunto $I$, mas apenas finitas vezes ao conjunto $W_{0}$, teremos que, se os tempos de retorno são $n_{1}, n_{2}, \ldots$, então

$$
\lim _{k \rightarrow \infty} \frac{1}{n_{k}} \sum_{i=0}^{n_{k}-1} \phi\left(\tilde{f}^{i}(x)\right) \leq \psi(q) .
$$

Como $T_{1}$ é homeomorfismo e cada ponto de $M$ tem finitas pré-imagens pela $f$, então cada ponto de $M$ tem finitas pré-imagens por $\tilde{f}$ (proposição 3.1.1). Ao considerar a transformação $T_{1}$ teremos uma vizinhança de $\alpha$,

$$
W_{\alpha}=W_{0} \bigcap f_{2}^{-1}\left(V\left(\overline{\alpha f_{2}(\alpha)}, \delta_{3}\right)\right)
$$




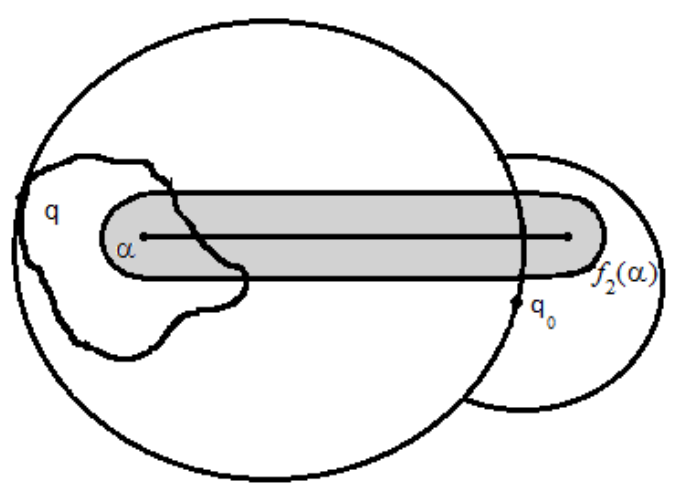

Figura 3.1: Vizinhança de $\overline{\alpha f_{2}(\alpha)}$.

Se $x \in W_{0} \backslash W_{\alpha}$ então $f_{2}(x) \in B\left[q_{0} ; \delta\right]$ e, em particular se $x \in D$, que retorna para $W_{0}$ no tempo $n_{1}$, podemos escrever $n_{1}=n_{x}+k \operatorname{com} f^{n_{x}}(x)=f_{2}(x) \in B\left[q_{0} ; \delta\right] \mathrm{e}$ $f^{n_{x}+k}(x) \in W_{0}$, ou seja, para voltar ao conjunto $W_{0}$ o ponto $x$ precisa passar primeiro pela vizinhança $B\left[q_{0} ; \delta\right]$ devido às construções dos conjuntos. Desta forma:

$$
\begin{aligned}
\frac{1}{n_{1}} \sum_{i=0}^{n_{1}-1} \phi\left(\tilde{f}^{i}(x)\right)-\psi(\alpha) & =\frac{n_{x} \psi(x)+k \psi\left(f_{2}(x)\right)}{n_{x}+k}-\psi(\alpha) \\
& \leq \frac{n_{x} \psi\left(x_{\max }\right)+k \psi(q)}{n_{x}+k}-\psi(\alpha) \\
& \leq \frac{n_{x} \psi\left(x_{\max }\right)+\psi(q)}{n_{x}+1}-\psi(\alpha) \\
& \leq \frac{n_{x}\left(\psi\left(x_{\max }\right)-\psi(\alpha)\right)-(\psi(\alpha)-\psi(q))}{n_{x}+1} \leq 0,
\end{aligned}
$$

onde a última desigualdade segue de (3.3).

De maneira análoga para infinitos retornos obtemos:

$$
\lim \frac{1}{n} \sum_{i=0}^{n-1} \phi\left(\tilde{f}^{i}(x)\right) \leq \psi(\alpha) .
$$

\subsubsection{A última perturbação}

Para completar a demostração do teorema (3.1.3) nos resta majorar as médias dos elementos que tem infinitos retornos no conjunto $W_{\alpha}$. A idéia para conseguir isso é 
considerar outra perturbação $T_{2}$ tal que, para o novo endomorfismo $T_{2} \circ \tilde{f}, \alpha$ seja um ponto repulsor e tal que a bacia de repulsão de $\alpha$ contenha $W_{\alpha}$. Como $\alpha$ será repulsor, toda órbita que tenha uma iterada futura em $W_{\alpha}$ e que não seja finalmente periódica, irá em algum tempo futuro, ter um retorno fora de $W_{\alpha}$. Buscaremos mostrar que, ainda que $\psi$ aplicada nos retornos desta órbita possa ser maior do que $\psi(\alpha)$, quando no futuro a órbita tiver um retorno fora de $W_{\alpha}, \psi$ aplicada neste retorno será suficientemente menor para que a média de Birkhoff global seja menor do que a do ponto periódico.

Definição 3.3.4. Seja $\tilde{D}=I \cap\left(\bigcup_{i=1}^{\infty} \tilde{f}^{-i}(I)\right)$ o conjunto dos pontos que voltam para I pela função $\tilde{f}$. Sobre esse conjunto definimos as funções:

$$
\begin{aligned}
\tilde{N}_{r e t}(x) & =\inf \left\{j \in \mathbb{N}^{*}: \tilde{f}^{j}(x) \in I\right\} \\
\tilde{f}_{2}(x) & =\tilde{f}^{\tilde{N}_{r e t}(x)}(x) \\
\tilde{\psi}(x) & =\frac{1}{\tilde{N}_{r e t}(x)} S_{\tilde{N}_{r e t}(x)} \tilde{f}(x)
\end{aligned}
$$

Proposição 3.3.5. As seguintes igualdades são verdadeiras:

a) $\tilde{D}=D$;

b) $\tilde{N}_{\text {ret }}(x)=N_{\text {ret }}(x)$ para todo $x \in \tilde{D}$;

c) $\tilde{\psi}(x)=\psi(x)$ para todo $x \in \tilde{D}$;

d) $\tilde{f}_{2}(x)=T_{1} \circ f_{2}(x)$ e se $x \notin f_{2}^{-1}\left(V\left(\overline{\alpha f_{2}(\alpha)}, \delta_{3}\right)\right)$, ent $\tilde{a} o \tilde{f}_{2}(x)=f_{2}(x)$.

\section{Demonstração.}

a) Vamos mostrar a dupla contenção. Primeiramente note que $\left.T_{1}\left(V\left(\overline{\alpha f_{2}(\alpha)}, \delta_{3}\right)\right)\right)=$ $V\left(\overline{\alpha f_{2}(\alpha)}, \delta_{3}\right)$ e que $T_{1}$ é a identidade fora deste conjunto e desta forma $T_{1}(I)=I$. Agora, seja $x \in D$, então existe $N_{\text {ret }}(x)>0$ tal que $f^{N_{r e t}(x)}(x) \in I$. Para $0<i<$ $N_{\text {ret }}(x)$ temos que $f^{i}(x) \notin I$, portanto $\tilde{f}^{i}(x)=f^{i}(x)$.

$$
\tilde{f}^{N_{r e t}(x)}(x)=T_{1}\left(f^{N_{r e t}(x)}(x)\right)=T_{1}(y) \in I,
$$

pois $y=f^{N_{r e t}(x)}(x) \in I, \operatorname{assim} D \subseteq \tilde{D}$. 
Se $x \in \tilde{D}$, por definição existe um $k>0$ tal que $\tilde{f}^{k}(x) \in I$. Suponha por absurdo que para todo $p>0, f^{p}(x) \notin I$. Como observado anteriormente, isto implica que $\tilde{f}^{p}(x)=f^{p}(x)$, para todo $p>0$, e em particular para $p=k$. Mas isso é absurdo, portanto $\tilde{D} \subseteq D$.

b) Fora de $I$ a função $T_{1}$ é a identidade, ou seja, $\tilde{f}=f$. Sejam $x \in D$ e $N_{\text {ret }}(x)$ seu tempo de primeiro retorno a $I$ pela $f$. Então $f^{N_{r e t}(x)-1}(x)=\tilde{f}^{N_{r e t}(x)-1}(x)$, pois $f^{i}(x) \notin$ $I$ para todo $0<i<N_{\text {ret }}(x)$. Aplicando novamente $\tilde{f}$ temos que $\tilde{N}_{\text {ret }}(x)=N_{\text {ret }}(x)$.

c) A função $T_{1}$ é a identidade fora de $I$. Como $f^{i}(x) \notin I$ para todo $0<i<N_{\text {ret }}(x)$ e $\tilde{N}_{r e t}(x)=N_{\text {ret }}(x)$, observe que

$$
\psi(x)=\frac{1}{N_{r e t}(x)} S_{N_{r e t}(x)} f(x)=\frac{1}{\tilde{N}_{r e t}(x)} S_{\tilde{N}_{r e t}(x)} \tilde{f}(x)=\tilde{\psi}(x),
$$

para todo $x \in \tilde{D}$.

d) A função $T_{1}$ é a identidade fora de $I$. Como $f^{i}(x) \notin I$ para todo $0<i<N_{\text {ret }}(x)$ e $\tilde{N}_{\text {ret }}(x)=N_{\text {ret }}(x)$,

$$
\tilde{f}_{2}(x)=\tilde{f}\left(\tilde{f}^{\tilde{N}_{r e t}(x)-1}(x)\right)=\left(T_{1} \circ f\right)\left(f^{N_{r e t}(x)-1}(x)\right)=T_{1} \circ f_{2}(x) .
$$

Agora, se $x \notin f_{2}^{-1}\left(V\left(\overline{\alpha f_{2}(\alpha)}, \delta_{3}\right)\right)$, temos que $f_{2}(x) \notin V\left(\overline{\alpha f_{2}(\alpha)}, \delta_{3}\right)$ e $T_{1} \circ f_{2}(x)=$ $f_{2}(x)$. Desta forma, $\tilde{f}_{2}(x)=f_{2}(x)$.

Seja $\psi_{\max }: I \rightarrow \mathbb{R}$ a seguinte função:

$$
\psi_{\max }(x)=\max _{y \in B[\alpha, d(\alpha, x)]} \psi(y) .
$$

Note que $\psi_{\max }(\alpha)=\psi(\alpha), \psi(x) \leq \psi_{\max }(x)$ e que se $d\left(\alpha, x_{1}\right)<d\left(\alpha, x_{2}\right)$ então $\psi_{\max }\left(x_{1}\right) \leq$ $\psi_{\max }\left(x_{2}\right)$.

Como $\psi_{\max }$ só depende da distância de $x$ a $\alpha$, assim podemos definir outra função $P: \mathbb{R} \rightarrow \mathbb{R}$ por

$$
P(s)=\sup _{x \in B(\alpha ; s)} \psi(x)-\psi(\alpha) .
$$

Esta função é contínua não-decrescente com $P(0)=0$, além disso, dado $x$ com $d(x, \alpha)=s$, então $P(s)=\psi_{\max }(x)-\psi(\alpha)$. 
Agora, definimos $R_{1}$ e $R_{2}$ reais positivos de maneira que $W_{\alpha} \subset B\left(\alpha ; R_{1}\right) \subset B\left(\alpha ; R_{2}\right)$ e $B\left(\alpha ; R_{2}\right) \subset B\left(x^{*}, \varepsilon^{*}\right)$ como na figura (3.2):

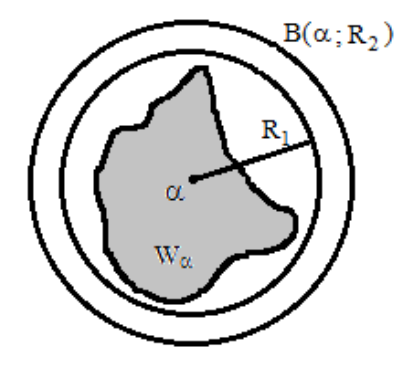

Figura 3.2: Região da perturbação.

Vamos fazer uma perturbação apenas em $B\left(\alpha ; R_{2}\right)$ da seguinte forma: defina para $0 \leq s \leq R_{1}$ o conjunto $A_{s}=\left\{x \in B\left(\alpha ; R_{1}\right): s \leq d(\alpha, x) \leq R_{1}\right\}$ e a função

$$
Q(s)=\inf _{x \in A_{s}} d\left(\tilde{f}_{2}(x), \alpha\right) .
$$

Observe que $Q(s)$ é não-decrescente e contínua. Além disso podemos assumir que $\alpha$ tem apenas uma pré-imagem pela $\tilde{f}_{2}$ em $B\left(\alpha ; R_{1}\right)$ pois, como notado anteriormente para $\tilde{f}_{2}$ todos os pontos tem um número finito de pré-imagens. Desta forma, $Q(0)=0$, e se $s>0$ então $Q(s)>0$.

Seja $s_{0}=R_{1}$, definimos duas sequências $s_{i}$ e $r_{i}$ pelas expressões:

$$
P\left(s_{i}\right)=\frac{1}{2^{i}} P\left(R_{1}\right) \text { e } r_{i}=\min \left\{Q\left(s_{i}\right), s_{i}\right\}
$$

Agora definimos uma função $\lambda(x)$ da seguinte maneira: Se $r_{k+1}<d(x, \alpha)<r_{k}<R_{1}$ então

$$
s_{k} \leq \lambda(x) d(x, \alpha) \leq s_{k-1}
$$

Uma função que satisfaz isso é por exemplo $\lambda(x)=\frac{s_{k}+\left(s_{k-1}-s_{k}\right) \frac{d(x, \alpha)-r_{k+1}}{r_{k}-r_{k+1}}}{d(x, \alpha)}$.

Se $d(x, \alpha)>R_{1}$ então $\lambda(x)=1$.

Definimos a perturbação $\hat{f}=T_{2} \circ \tilde{f}$, onde $T_{2}$ restrita ao complementar de $B\left(\alpha ; R_{2}\right)$ é a identidade, e restrita a $B\left(\alpha ; R_{1}\right)$ é definida por

$$
T_{2}(x)=\lambda(x)(x-\alpha)+\alpha,
$$


que é uma expansão radial, com velocidade variável $\lambda(x)$. A função $T_{2}$ assim definida é contínua. Seja $\hat{f}_{2}(x)=T_{2} \circ \tilde{f}_{2}(x)$, temos o seguinte lema.

Lema 3.3.6. Se $x \in W_{\alpha} \operatorname{com} d\left(\hat{f}_{2}(x), \alpha\right)<s_{k}$ então:

$$
d(x, \alpha) \leq s_{k+1}
$$

Demonstração. Se $d(x, \alpha)>s_{k+1}$ então $d\left(\tilde{f}_{2}(x), \alpha\right)>r_{k+1}$ o que implica

$$
d\left(\hat{f}_{2}(x), \alpha\right)=d\left(\lambda\left(\tilde{f}_{2}(x)\right)\left(\tilde{f}_{2}(x)-\alpha\right)+\alpha, \alpha\right)=\lambda\left(\tilde{f}_{2}(x)\right) d\left(\tilde{f}_{2}(x), \alpha\right) \geq s_{k},
$$

desta maneira concluímos que, se $d\left(\hat{f}_{2}(x), \alpha\right)<s_{k}$, então $d(x, \alpha) \leq s_{k+1}$.

Proposição 3.3.7. Se $x, \hat{f}_{2}(x), \ldots, \hat{f}_{2}^{N}(x) \in W_{\alpha}$, então $d(x, \alpha) \leq s_{N}$.

Demonstração. Vamos demonstrar a proposição por indução sobre $N$, para $N=0$ temos que $d(x, \alpha) \leq R_{1}$ portanto $d(x, \alpha) \leq s_{0}$, supomos que vale para $k=N-1$.

Para $k=N$, seja $y=\hat{f}_{2}(x)$ como $y, \hat{f}_{2}(y), \ldots, \hat{f}_{2}^{N-1}(y) \in W_{\alpha}$ então pela hipótese de indução:

$$
d(y, \alpha) \leq s_{N-1} \Rightarrow d\left(\hat{f}_{2}(x), \alpha\right) \leq s_{N-1} \text { pelo lema }(3.3 .6) d(x, \alpha) \leq s_{N} .
$$

Lema 3.3.8. Se $x, \hat{f}_{2}(x), \ldots, \hat{f}_{2}^{k}(x) \in W_{\alpha}$, então $N_{\text {ret }}\left(\hat{f}_{2}^{i}(x)\right)=N_{\text {ret }}(q)$ para todo $i=1, \ldots, k e$

$$
\frac{1}{k N_{\text {ret }}(q)} S_{k N_{r e t}(q)} \hat{f}(x) \leq \psi(\alpha)+\frac{1}{k}\left(\psi\left(x_{\max }\right)-\psi(\alpha)\right)
$$

Demonstração. Da maneira como definido $W_{\alpha}$, temos que, se $x, \hat{f}_{2}(x), \ldots, \hat{f}_{2}^{k}(x) \in$ $W_{\alpha}$, então $N_{\text {ret }}\left(\hat{f}_{2}^{i}(x)\right)=N_{\text {ret }}(q)$ para todo $i=1, \ldots, k$. Agora note que

$$
\begin{aligned}
\frac{1}{k N_{r e t}(q)} S_{k N_{r e t}(q)} \hat{f}(x) & =\frac{1}{k N_{r e t}(q)} \sum_{i=0}^{k-1} N_{r e t}(q) \psi\left(\hat{f}_{2}^{i}(x)\right) \\
& \leq \frac{1}{k} \sum_{i=0}^{k-1} \psi_{\max }\left(\hat{f}_{2}^{i}(x)\right) \text { e como } P(d(x, \alpha))=\psi_{\max }(x)-\psi(\alpha) \\
& \leq \psi(\alpha)+\frac{1}{k} \sum_{i=0}^{k-1} P\left(d\left(\hat{f}_{2}^{i}(x), \alpha\right)\right) .
\end{aligned}
$$


Pela proposição $(3.3 .7) d\left(\hat{f}_{2}^{i}(x), \alpha\right) \leq s_{k-i}$, assim

$$
P\left(d\left(\hat{f}_{2}^{i}(x), \alpha\right)\right) \leq P\left(s_{k-i}\right)=\frac{1}{2^{k-i}} P\left(R_{1}\right),
$$

além disso, $P\left(R_{1}\right) \leq \psi\left(x_{\max }\right)-\psi(\alpha)$. Desta forma concluímos que:

$$
\begin{aligned}
\frac{1}{k} \sum_{i=0}^{k-1} P\left(d\left(\hat{f}_{2}^{i}(x), \alpha\right)\right) & \leq \frac{1}{k}\left(\frac{1}{2^{k}}+\frac{1}{2^{k-1}}+\ldots+\frac{1}{2}\right) P\left(R_{1}\right) \\
& \leq \frac{1}{k} P\left(R_{1}\right) \leq \frac{1}{k}\left(\psi\left(x_{\max }\right)-\psi(\alpha)\right)
\end{aligned}
$$

e temos o resultado.

Assim, dado $x \in W_{\alpha}$ que retorna infinitas vezes para este conjunto, e seja $n_{j}$ a sequência dos tempos tais que $\hat{f}^{n_{j}}(x)=\left(T_{2} \circ \tilde{f}\right)^{n_{j}}(x) \in I$, onde $n_{0}=0$ e $n_{i+1}=$ $n_{i}+N_{\text {ret }}\left(\hat{f}^{n_{i}}(x)\right)$ e considere as seguintes subsequências de $\left(n_{k}\right)_{k \in \mathbb{N}}$ :

- $a_{j}$, onde $a_{1}=0$ e $a_{i+1}$ é o menor inteiro maior que $a_{i}$ tal que $\hat{f}^{n_{a_{i+1}}}(x)$ está em $W_{\alpha}$, mas $\hat{f}^{n_{a_{i+1}-1}}(x)$ não está.

- $b_{j}$, onde $b_{i}$ é o menor inteiro maior que $a_{i}$ tal que $\hat{f}^{n_{b_{i}-1}}(x)$ está em $W_{\alpha}$, mas $\hat{f}^{n_{b_{i}}}(x)$ não está.

Utilizando estas sequências temos:

$$
\begin{array}{r}
\frac{1}{n_{a_{k}}} S_{n_{a_{k}}} \hat{f}(x)=\frac{1}{n_{a_{k}}} \sum_{l=0}^{a_{k}-1} N_{r e t}\left(\hat{f}^{n_{l}}(x)\right) \psi\left(\hat{f}^{n_{l}}(x)\right) \\
=\frac{1}{n_{a_{k}}} \sum_{j=1}^{k-1}\left(\sum_{l=a_{j}}^{b_{j}-1} N_{r e t}\left(\hat{f}^{n_{l}}(x)\right) \psi\left(\hat{f}^{n_{l}}(x)\right)+\sum_{l=b_{j}}^{a_{j+1}-1} N_{r e t}\left(\hat{f}^{n_{l}}(x)\right) \psi\left(\hat{f}^{n_{l}}(x)\right)\right),
\end{array}
$$

além disso se $a_{j} \leq i \leq b_{j}-1$ então $\hat{f}^{n_{i}}(x) \in W_{\alpha}$ e se $b_{j} \leq i \leq a_{j+1}-1$ então $\hat{f}^{n_{i}}(x) \notin W_{\alpha}$, para todo $x \in W_{\alpha}$, desta forma:

$$
\sum_{l=b_{j}}^{a_{j+1}-1} N_{r e t}\left(\hat{f}^{n_{l}}(x)\right) \psi\left(\hat{f}^{n_{l}}(x)\right) \leq\left(n_{a_{j+1}}-n_{b_{j}}\right) \psi(q)
$$


Para o outro termo da expressão (3.5) temos:

$$
\begin{aligned}
& \sum_{l=a_{j}}^{b_{j}-1} N_{r e t}\left(\hat{f}^{n_{l}}(x)\right)\left(\psi\left(\hat{f}^{n_{l}}(x)\right)-\psi(\alpha)\right)=\sum_{l=a_{j}}^{b_{j}-1} N_{r e t}(q) \psi\left(\hat{f}^{n_{l}}(x)\right)-\left(b_{j}-a_{j}\right) N_{\text {ret }}(q) \psi(\alpha) \\
\leq & \left(b_{j}-a_{j}\right) N_{\text {ret }}(q)\left(\psi(\alpha)+\frac{1}{b_{j}-a_{j}}\left[\psi\left(x_{\max }\right)-\psi(\alpha)\right]\right)-\left(b_{j}-a_{j}\right) N_{r e t}(q) \psi(\alpha) \\
= & N_{\text {ret }}(q)\left(\psi\left(x_{\max }\right)-\psi(\alpha)\right) \leq \frac{\psi(\alpha)-\psi(q)}{2}
\end{aligned}
$$

isto é verdade pela desigualdade do lema(3.3.8) e por (3.3), já que $N_{\text {ret }}(q)<m_{0}$. Portanto

$$
\sum_{l=a_{j}}^{b_{j}-1} N_{r e t}\left(\hat{f}^{n_{l}}(x)\right) \psi\left(\hat{f}^{n_{l}}(x)\right) \leq \frac{\psi(\alpha)-\psi(q)}{2}+\left(n_{b_{j}}-n_{a_{j}}\right) \psi(\alpha) .
$$

Substituindo (3.6) e (3.7) em (3.5) resulta:

$$
\frac{1}{n_{a_{k}}} \sum_{j=1}^{k-1}\left[\psi(\alpha)\left(n_{b_{j}}-n_{a_{j}}-\frac{1}{2}\right)+\psi(q)\left(n_{a_{j+1}}-n_{b_{j}}+\frac{1}{2}\right)\right]
$$

da maneira como foi escolhido $\alpha$ vale a desigualdade $\psi(\alpha) \geq \psi(q)$, assim

$$
\begin{gathered}
\frac{1}{n_{a_{k}}} \sum_{j=1}^{k-1}\left[\psi(\alpha)\left(n_{b_{j}}-n_{a_{j}}-\frac{1}{2}\right)+\psi(q)\left(n_{a_{j+1}}-n_{b_{j}}+\frac{1}{2}\right)\right] \\
\quad \leq \frac{1}{n_{a_{k}}} \sum_{j=1}^{k-1} \psi(\alpha)\left(n_{a_{j+1}}-n_{a_{j}}\right)=\psi(\alpha)-\frac{n_{a_{1}}}{n_{a_{k}}} \psi(\alpha) \rightarrow \psi(\alpha) .
\end{gathered}
$$

O que conclui a demonstração do teorema (3.1.3).

\subsection{Problemas Futuros}

Uma pergunta natural surge ao provarmos o teorema (3.1.3): Ter órbita periódica maximizante vale em um conjunto genérico de endomorfismos? A resposta é negativa e está no trabalho [1], no qual os autores mostraram a relação entre a regularidade da $\phi$ 
e a generecidade. Existem outras questões em aberto, por exemplo, sobre a velocidade de aproximação dos endomorfismos que possuem medida maximizante suportada em órbita periódica. No trabalho [34] os autores ao considerarem a dinâmica do sub-shift de tipo finito e um potencial $\phi$ Hölder contínuo, estudaram a velocidade de aproximação de $\int \phi d \mu$, por medidas com período menores que $p$. Outra possível questão é estudar o problema para endomorfismos com mais regularidade como por exemplo, trocar $C^{0}$ por $\alpha$-Hölder:

Conjectura 3.4.1. Seja X uma variedade Riemanianna compacta, então o conjuntos dos endomorfismos $\alpha$-Hölder contínuos definidos sobre $X$, que possuem uma medida $\phi$-maximizante suportada em uma órbita periódica é denso no conjunto dos endomorfismos $\alpha$-Hölder contínuos nesta topologia.

Outro problema a ser estudado é o de considerar $X$ não compacto. Jenkinson et. al em [20] determinaram que condições $\phi$ deve satisfazer para garantir a existência de medidas $\phi$-maximizantes. Quando o espaço não é compacto podemos não ter medidas borelianas de probabilidade invariantes por uma dinâmica, e mesmo que tenhamos podemos não ter medidas maximizantes, por exemplo: Seja o espaço $X=\mathbb{Z}$ e a dinâmica $f: X \rightarrow X$ dada por $f(n)=n-1$, então $\mathcal{M}_{f}(X)=\emptyset$. Mas se considerarmos $X=\mathbb{R}$ e a dinâmica $f(x)=x, \mathcal{M}_{f}(X) \neq \emptyset$ e ainda assim, o conjunto das medidas $\phi$-maximizantes para $\phi(x)=\arctan (x)$ é vazio.

Definição 3.4.2. Seja $Y$ um espaço topológico. Denotemos por $C B(Y)$ o conjunto das funções contínuas a valores reais limitadas em $Y$ e $f: Y \rightarrow Y$ uma aplicação contínua. Uma função da forma $\phi-\phi \circ f$, onde $\phi \in C B(Y)$, é chamada um cobordo. Duas funções $g$ e $h$ que diferem por uma cobordo são chamadas cohomólogas. Uma função contínua $\tilde{\xi}$ cohomóloga a $\xi$ é chamada uma forma normal para $\xi$ se

$$
\tilde{\xi}^{-1}(\sup \tilde{\xi})
$$

contém o suporte de alguma medida de probabilidade $f$-invariante. 
Lema 3.4.3. Suponha $f: X \rightarrow X$ uma função contínua sobre o espaço topológico $X$, e que a função contínua $\phi: X \rightarrow \mathbb{R}$ tenha uma forma normal $\tilde{\phi}$, então:

$$
\mathcal{M}_{\phi-\max }(X)=\left\{\mu \in \mathcal{M}_{f}: \operatorname{supp}(\mu) \subset \tilde{\phi}^{-1}(\sup \tilde{\phi})\right\} \neq \emptyset
$$

O problema para garantir a existência de medidas $\phi$-maximizantes se resume a encontrar formas normais para $\phi$, porém nem toda função possui uma forma normal, mesmo que ela seja contínua e definida sobre um espaço compacto [4].

Definição 3.4.4. Seja $f: X \rightarrow X$ um endomorfismo sobre o espaço topológico $X$. Uma função contínua $\phi: X \rightarrow \mathbb{R}$ é dita essencialmente compacta (em relação a $f$ ) se existe $\xi \in C B(X)$ onde $\xi(x)=\sup _{y \in f^{-1}(x)}(\phi-\lambda(\phi)+\xi)(y)$, onde

$$
\lambda(\phi)=\lim _{n \rightarrow \infty} \frac{1}{n} \sup _{x \in X} \sum_{i=0}^{n-1}(f \circ \phi)(x),
$$

e existe um conjunto $Y \subset X$ tal que:

(a) $\bigcap_{n=0}^{\infty} f^{-n} Y$ é não vazio e compacto,

(b) $f(Y)=X$

(c) para cada $x \in X$,

$$
\xi(x)+\lambda(\phi)=\sup _{y \in f^{-1}(x) \cap Y}(\phi+\xi)(y)
$$

Definição 3.4.5. Um espaço topológico $X$ é chamado de espaço polonês se ele for separável, metrizável e completo em relação a métrica.

Usando as definições acima O. Jenkinson demonstrou o seguinte teorema:

Teorema 3.4.6. Seja $f: X \rightarrow X$ um endomorfismo sobre um espaço Polonês $X$. Se a função contínua $\phi: X \rightarrow \mathbb{R}$ é essencialmente compacta então:

$$
\mathcal{M}_{\phi-\max }(X)=\left\{\mu \in \mathcal{M}_{f}: \operatorname{supp}(\mu) \subset \tilde{\phi}^{-1}(\sup \tilde{\phi})\right\} \neq \emptyset
$$

Como consequência deste teorema e do nosso resultado temos a seguinte conjectura: 
Conjectura 3.4.7. Seja X uma variedade Riemanianna não necessariamente compacta e $\phi: X \rightarrow \mathbb{R}$ essencialmente compacta, então o conjunto dos endomorfismos sobre $X$ que possuem uma medida $\phi$-maximizante é denso no conjunto de endomorfismos.

Observação: Como $\phi$ é essencialmente compacta, temos pelo resultado de Jenkinson que o conjunto das medidas $\phi$-maximizantes é não vazio. Note que, o teorema da decomposição ergódica é valido mesmo quando o $X$ não é compacto, basta que o espaço das medidas borelianas de probabilidade seja não vazio [14], assim podemos tomar $\mu_{\max }$ ergódica, com $\int \phi d \mu_{\max }=0$. No entanto, para refazer a construção da prova do teorema (3.1.3), precisamos de um análogo à proposição (3.1.6), pois sabemos que ela não é verdadeira em espaços não compactos [4]. 

[1] ADDAS-ZANATA, S. e TAL, F. A., Support of maximizing measures for typical $C^{0}$ dynamics on compact manifolds, Discrete and Continuous Dynamical Systems, 26 vol 3 (2010), 785-804.

[2] ATKInSON, G., Recurrence of co-cycles and random walks J. London Math. Soc., s2-13 (1976), 486-488.

[3] BARAVIERA, A. T., LEPlaideur, R., LOPES, A. O., Selection of measures for a potential with two maxima at the zero temperature limit. preprint, (2010), $1-21$.

[4] BATISTA, T. C., Alguns resultados sobre otimização ergódica em espaços não compactos. Dissertação de Mestrado, São Paulo, (2009).

[5] BISSACOT, R. e GARIBALDI, E. Weak KAM methods and ergodic optimal problems for countable Markov shifts, Bull. of the Brazilian Mathematical Society, 41 (2010), 321-338.

[6] BISSACOT, R. e FREIRE Jr., R. S., On the existence of maximizing measures for irrducible countable Markov shifts: a dynamical proof. ... 
[7] BOUSCH, T., Le poisson n'a pas d'arête, Ann. Inst. Henri Poicaré, 36 (2000), 489-508.

[8] BOUSCH, T., La condition de Walters, Ann. scientifiques de l'Ecole Normale Supérieure, 34 (2001), 287-311.

[9] BOUSCH, T. e JENKINSON, O., Cohomology classes of dynamically non-negative $C^{k}$ functions, Inventiones Mathematicae, 148 (2002), 207-217.

[10] BRANTON, S. D., Sub-actions for Young towers, Discrete e Continuous Dynamical Systems, Series A, 22 (2008), 541-556.

[11] BRÉMONT, J., On the behaviour of Gibbs measures at temperature zero, Nonlinearity, 16 (2003), 419-426.

[12] CONTRERAS, G. e ITURRIAGA, R., Global minimizers of autonomous Lagrangians, http://www.cimat.mx/ gonzalo/, (2000).

[13] CONZE, J.-P. e GUIVARC'H, Y., Croissance des sommes ergodiques et principe variationnel, Relatório Técnico - Université de Rennes I (1993), 1-29.

[14] Deuschel, J. D. e STROOCK, D. W., Large Deviations, London, Academic Press, (1989).

[15] GARIBALDI, E., Introduction to expanding ergodic optimization, Notas de aula, UNICAMP - SP, 2009.

[16] GARIBALDI, E., LOPES, A. O. e THIEUlLEN, Ph., On calibrated and separating sub-actions, Bull. Soc. Bras. Math. Vol 404 (2009), 587-612.

[17] GaRIBAlDI, E., THIEUlLEN, Ph., Minimizing orbits in the discrete AubryMather model, Nonlinearity 24, (2011), 563-611

[18] JENKINSON, O., Ergodic optimization, Discrete and Continuous Dynamical Systems, 15 (2006), 197-224. 
[19] JENKINSON, O., MAULDIN, R. D. e URBAŃSKI, M., Ergodic optimization for countable alphabet subshifts of finite type, Ergodic Theory and Dynamical Systems, 26 (2006), 1791-1803.

[20] JENKINSON, O., MAULDIN, R. D. e URBAŃSKI, M., Ergodic optimization for noncompact dynamical systems, Dynamical Systems, 22 (2007), 379-388.

[21] JENKINSON, O., Every ergodic measure is uniquely maximizing, Discrete and Continuous Dynamical Systems, 16 (2006), 383-392.

[22] JENKINSON, O. MAULDIN, R. D. e URBAŃSKI, M., Zero temperature limits of Gibbs equilibrium states for countable alphabet subshifts of finite type, J. Stat. Phys., 119 (2005), 765-776.

[23] JENKINSON, O., A partial order on 2x-invariant measures, Math. Res. Lett., 15 (2008), 100.01-08.

[24] KATOK, A., HASSELBLATT, B., Introduction to the Modern Theory of Dynamical Systems, Cambridge University Press, (2006).

[25] LOPES, A. O. e THIEUllEN, Ph., Sub-actions for Anosov diffeomorfisms, Astérisque, 287 (2003), 135-146.

[26] LOPES, A. O. e THIEUlLEN, Ph., Sub-actions for Anosov flows, Ergodic Theory and Dynamical Systems, 25 (2005), 605-628.

[27] LOPES, A. O., CONTRERAS, G. and THIEUllEN, Ph, Lyapunov minimizing measures for expanding maps of the circle. Ergodic Theory and Dynamical Systems, 21 (2001), 1379-1409.

[28] MAÑÉ, R., On the minimizing measures of Lagrangian dynamical systems, Nonlinearity, 5 no. 3 (1992), 623-638.

[29] MAÑÉ, R., Generic properties and problems of minimizing measures of Lagrangian systems, Nonlinearity, 9 (1996), 273-310. 
[30] MASSART, D., On Mañé's last conjecture, (2002), 1-6.

[31] MATHER, J., Action minimizing invariant measures for positive definite Lagrangian systems, Math Z., 207 (1991), 169-207.

[32] MATHER, J., Minimal measures, Comment. Math. Helv., 64 no. 3 (1989), 375394.

[33] MILnOR, John W., Collected Works Vol. III, Differential Topology, American Mathematical Society (2007).

[34] MORRIS, I. D., A sufficient condition for the subordination principle in ergodic optimization, Bul. London Math. Soc., 39 (2007), 214-220.

[35] QUAS, A. e SIEFKEN, J., Ergodic optimization of super-continuous functions in the shift, arXiv.1101.2706v1 [math.DS] 14 Jan_2011, (2011), 1-24.

[36] ROCKAFELLAR, R. T., Extension of Fenchel's duality theorem for convex functions, Duke Mathematical Journal, 33 (1966), 81-89.

[37] RUDIN, W., Real and complex analysis, New York, McGraw-Hill (1987).

[38] RUELlE, D., Thermodynamic Formalism, Cambridge University Press, 2 ed., (2004)

[39] SOUZA, R. R., Sub-actions for weakly hyperbolic one-dimensional systems, Dynamical Systems, 18 (2003), 165-179.

[40] YANG, T-H., HUNT, B. R. and OTT, E., Optimal periodic orbits of continuous time chaotic systems. Phys. Rev. E, 62(2) (2000), 1950-1959.

[41] TAL, F. A. e ADDAS-ZANATA, S., Maximizing measures for endomorphisms of the circle, Nonlinearity, 21 (2008), 2347-2359.

[42] TAL, F. A. e ADDAS-ZANATA, S., On maximizing measures of homeomorphisms on compact manifolds, Fundam. Math., 200 (2008), 145-159. 
[43] WALTERS, P., An introduction to Ergodic Theory, Springer-Verlag, New York (2000). 